\title{
Asymmetric Information and Survival in Financial Markets
}

\author{
Emanuela Sciubba* \\ Faculty of Economics and Politics \\ University of Cambridge
}

\begin{abstract}
In the evolutionary setting for a financial market developed by Blume and Easley (1992), we consider an infinitely repeated version of a model à la Grossman and Stiglitz (1980) with asymmetrically informed traders. Informed traders observe the realisation of a payoff relevant signal before making their portfolio decisions. Uninformed traders do not have direct access to this kind of information, but can partially infer it from market prices. As a counterpart for their privileged information, informed traders pay a per period cost. As a result, information acquisition triggers a trade-off in our setting. We prove that, as long as information is costly, a strictly positive measure of uninformed traders will survive. This result contributes to the literature on noise trading. It suggests that Friedman (1953)'s argument against the importance of noise traders in the process of price determination is too simplistic. Traders whose beliefs are "wrong" according to the best available information, in fact, are not wiped out by market forces and do affect asset prices in the long run.
\end{abstract}

JEL Classification: D50, D82, G14.

Keywords: Evolution; Portfolio Rules; Partially Revealing REE; Noise Trading.

Address for Correspondence: Emanuela Sciubba, Lucy Cavendish College, CB3 0BU Cambridge, UK. E-mail: es204@econ.cam.ac.uk; http:\\www.cus.cam.ac.uk 〜 es204.

\section{Introduction}

\subsection{Motivation}

There is very little doubt that superior information is the key to the golden world of moneymaking in financial markets. Who ever is not after superior information? Individual investors, who would typically hold a single stock or a small number of stocks, pick them through their own research or on the advice of financial press or private bankers. Fund managers - who often would not follow economists' advice to entrust their clients' resources to the market portfolio - do stock-picking on the basis of their best information. Banks

* I am deeply indebted to Luca Anderlini for his helpful guidance. I also benefited from discussion with Jayasri Dutta and Thorsten Hens. Useful comments came from participants to seminars in Bielefeld and Erasmus University, to the "PhD Awards" Italian tour in Rome, Naples, Padova and Milan, and to the "Shadow Talk" in Cambridge. All remaining errors are mine. 
proudly boast their research departments, to convince clients that their traders possess the best insight into economic conditions and into the financial analysis of individual companies.

The common belief is that well informed traders will make money at the expenses of the uninformed. The aim of this paper is to assess whether this statement is always true: in particular we ask to what extent it is valid when information acquisition is a costly activity.

Research is expensive: only sizeable banks can afford full-fledged economic research departments. Individual investors acquire information buying and reading newspapers and specialised press, tracing and analysing companies' balance sheets, and the like. Nobody who has gone through one of these readings at least once would doubt that those are costly activities. Alternatively, investors can buy information through a private banker who will be more than happy to sell them his expertise at a high fee. Who does get information for free? Even when individuals profit from information as insiders, the cost of - if not obtaining - at least using that information for trading purposes, is in the threat of a lawsuit for insider trading.

What we are trying to imply here is that, if it is true that superior information is often seen as an essential feature of money-making in financial markets, it is also true that the acquisition of superior information is - and is recognised as - a costly activity.

We consider a stylised model of a financial market where there are two types of traders: traders who invest in costly information acquisition and traders who take portfolio decisions looking only at the prices of assets.

The focus of the paper is twofold. First, we are interested in the characterisation of a non fully revealing informational equilibrium in a dynamic setting. In particular, we examine the process of information revelation through market prices as the population shares of informed and uninformed traders vary in time, in an infinitely repeated version of a model à la Grossman and Stiglitz [21].

Second, we are interested in the long run market outcome. In particular we ask what is the asymptotic population share of uninformed traders. Our results suggest that, as long as information is costly, uninformed traders are not driven to extinction, even when prices do not fully reveal the information possessed by informed traders. We believe that this result can constitute a valuable contribution to the literature on noise trading.

Uninformed traders in our setting trade on the basis of the information revealed by market prices. They can approximate but never attain the information possessed by informed traders. As a result, they take portfolio decisions which are "wrong" according to the best available information. For this reason we claim that uninformed traders in our model trade, at least partially, on noise rather than on information.

The debate on noise trading is quite lively in financial economics. Noise trading is essential to the existence of liquid markets, however it is difficult to find a good economic reason for the existence of noise traders. If noise traders are known to lose money to the informed, then why would uninformed individuals trade in the first place? As Fischer Black [17] puts it: "Noise trading provides the essential missing ingredient [for a liquid financial market]. Noise trading is trading on noise as if it were information. People who trade on noise are willing to trade even though from an objective point of view they would be better off not trading. Perhaps they think that the noise they are trading on is information. Or perhaps they just like to trade." ([17], p. 531). 
As a result, noise traders have been seen by the literature either as irrational investors or as agents who gain utility by their trading activity. However, even when one postulates one of these two "motivations" to trade, another major difficulty in accounting for the presence of noise traders is the widely accepted argument against their importance in the process of price formation. This point has been forcefully made by Friedman [18]. In his own words, the argument that speculation by noise traders is "destabilising [...] is largely equivalent to saying that speculators lose money, since speculation can be destabilising in general only if speculators on [...] average sell [...] low [...] and buy [...] high" ([18], p. 175). As a result, even if noise traders affect market prices, they will not do it for long, as they will be wiped out by market forces. Even if we believe that noise traders have a good reason to exist in the first place, we still have to account for their persistence: economic arguments, in fact, suggest that they should go bankrupt and disappear from the market.

Our results show that Friedman's argument, though intuitively appealing, is incomplete, since noise traders survive and affect asset prices in the long run.

\subsection{Related Literature}

There are at least four distinct streams of literature related to the present paper. First, for the type of model we use, this paper is related to the literature on evolution and market behaviour. Second, the paper specifically contributes to the literature on noise trading. Third, because of the equilibrium concept we use, the paper is nested in the rich literature on partially revealing rational expectations equilibria. Finally, as a general motivation for this study, the paper is related to the literature that looks at the emergence of imitative behaviour in a world where gathering information is a costly activity.

The literature on evolution and market behaviour is in its relative infancy. The idea that economic institutions can be modelled as biological organisms is not new, and evolutionary ideas such as natural selection and adaptive behaviour have had a long history in economic analysis. In recent years, the research effort in this direction has become so impressive and diverse that we will not attempt a review here. We will, instead, concentrate on the more self-contained strand of literature on which the present paper builds. In particular, we will focus on the literature that aims at studying long run market outcomes as the result of a process akin to natural selection, in which population dynamics are endogenous and emerge from the process of wealth accumulation. A seminal study in this direction is Blume and Easley [6]. They develop an evolutionary model of a financial market and identify conditions for survival. In particular, they show that the fittest behaviour in a risky environment is prescribed by a logarithmic utility function. Namely, whenever a logarithmic utility maximiser enters the market, all other traders are driven to extinction unless they asymptotically behave as if they were logarithmic utility maximisers ${ }^{1}$.

In this paper, we adopt Blume and Easley's definition of dominance, survival and extinction of traders. However our framework differs from theirs for two main reasons. First, we introduce signals and asymmetric information in their simpler setting. Second, in the present model markets are incomplete. We introduce incompleteness in a model à la Blume and Easley, through a random vector of asset supplies. Properties of the logarithmic utility

\footnotetext{
${ }^{1}$ Sciubba [34] shows that the same fate awaits CAPM traders and mean-variance traders.
} 
function guarantee, however, that logarithmic utility maximisation is still the fittest behaviour among traders with the same information sets. For this reason, we assume that all traders are logarithmic utility maximisers, so that we can concentrate on the fitness of their beliefs rather than portfolio rules.

Sandroni [33] addresses a similar problem: he looks at the fitness of beliefs and shows that, when agents choose savings endogenously, the most prosperous traders are those who are able to make the most accurate predictions ${ }^{2}$. The main intuition behind his result is that traders who also choose savings rates will allocate more wealth to future states of nature that they believe most likely to occur. This feature makes their behaviour "closer" to logarithmic utility maximisation. As a result, among agents displaying equally fit portfolio rules, those with the most accurate predictions will dominate.

The literature on noise trading, in recent years, has drawn many ideas from the literature on evolution and market behaviour ${ }^{3}$. Shefrin and Statman [35] develop a model in which sophisticated and informed traders interact with traders who make systematic cognitive errors. They show that, provided that noise traders are patient enough and that they do not commit errors which are "too serious", they will not be driven to extinction by informed traders. In our paper, population dynamics do not hinge on savings behaviour, but exclusively on the adopted policy for information acquisition.

Bias and Shadur [3] consider a financial market where buyers and sellers are randomly matched in a situation of bilateral monopoly and trade to share risk. They show that irrational traders who misperceive risk can, in fact, enjoy a higher bargaining power, so that they can obtain larger gains from trade than rational agents. In their setting, only two dynamically stable equilibria are possible: one in which there are rational sellers and irrational buyers and one in which there are irrational sellers and rational buyers. Unlike us, they find no stable equilibrium where rational and irrational players coexist on the same side of the market.

Kyle and Wang [27] consider a duopolistic market where a single risky asset is traded and examine the survival of traders who make mistakes in assessing the precision of their informative signals. They show that overconfidence acts like a commitment device in a Cournot duopoly model, "giving the overconfident trader a reputation for trading so aggressively that his rational opponent (recognizing this) trades less aggressively" ([27], p. 2074); as a result, overconfident traders may enjoy higher profits and survive in the long run. In the present paper, we argue that traders with "wrong" beliefs survive also in a competitive market setting, where the portfolio choice is not strategic.

De Long et al. [11][12] consider the long run distribution of wealth between rational investors and noise traders. They show that noise traders can eventually come to dominate the market if they unwillingly happen to make "good" cognitive mistakes. In fact, they can exhibit a faster rate of wealth accumulation if their misperceptions cause them to mimic logarithmic utility maximisers. They argue that there is a large class of plausible

\footnotetext{
${ }^{2}$ From this perspective, both Sandroni [33] and the present paper, also relate to the literature which studies converge to rational expectations. See, for instance, the three related papers by Blume et al [4], Bray [8] and Blume and Easley [5].

${ }^{3}$ Noise trading has been extensively studied also from a welfare perspective. See for instance, Stein [37] that shows how speculation reduces the informativeness of market prices and therefore causes a welfare loss.
} 
misperceptions that would lead to this result.

In their model, though, noise traders do not affect prices. In their own words, they "take a first step in considering the long-run distribution of wealth and examine a model in which noise traders do not affect prices. If they did affect prices, the returns on assets would depend on the distribution of wealth between noise traders and rational investors. This added complication would make analytical solutions [...] very difficult" ([12], p. 2).

In the present paper, uninformed traders do affect market prices and we believe that this is our main contribution to the literature on noise trading.

This paper is very close in spirit to Grossman and Stiglitz [21] and can, in fact, be viewed as an infinitely repeated version of their model. Grossman and Stiglitz consider a static financial market where there are two types of individuals: those who observe a signal which gives noisy information on the return of the risky asset and those who only observe the market price and infer information from it ${ }^{4}$. Their main contribution is to show that as long as information is costly, everyone informed or everyone uninformed is not an equilibrium.

Like Grossman and Stiglitz, our model is in the tradition of the noisy rational expectations model introduced by Lucas [29]. The main feature, and difficulty, of this type of models lies in the fact that the function served by prices is not only market clearing, but also information transmission. A well-known result is that, generically, when there are finitely many states of private information, there exists a rational expectation equilibrium (REE) and all the REE are fully revealing. Fxistence of partially revealing equilibria is more problematic and general results are not known. Consequently, in the literature existence is proved for parametric models where utilities are negative exponential and disturbances are normal. See, for example: Kreps [26], Grossman [20], Green [19], Hellwig [22], Grossman and Stiglitz [21], Diamond and Verrecchia [13], Bray [7], Verrecchia [38], Dow and Rahi [14].

In this paper we assume that all traders display the "fittest" long run behaviour towards risk and are therefore endowed with logarithmic utility functions. Under this assumption, existence of a partially revealing equilibrium is guaranteed in our setting.

Finally, the main result in this paper is related to the literature on the evolutionary emergence of imitative behaviour. In particular, Colinsk [9] develops an evolutionary model where in the long run costly optimisers coexist with cheap imitators. Finally, Rogers [31] examines the trade-off between social and individual learning. He shows that a stable equilibrium is attained at a level of learning such that the fitness of social learning is the same as of individual learning and he argues that in equilibrium individual learning is not maximised, so that indeed free-riding on information acquisition prevents the achievement of a fully efficient outcome.

\footnotetext{
${ }^{4}$ For experimental evidence supporting [21], see Sunder [36].

${ }^{5}$ The study of the generic existence of REE has concentrated on three cases, according to whether the dimension of the space of "states of private information" is less than, equal to, or greater than the price space. In the first case, Radner [30] and Allen [1] prove that fully revealing equilibria exist generically, i.e. except for a measure-zero set of economies. In the second case existence of fully revealing equilibria can obtain, but not generically. Finally, in the third case, Jordan [24] shows that there is a set of economies which permit equilibria arbitrarily close to full revelation. For a survey, see Jordan and Radner [25]. Also, for welfare analysis of rational expectations equilibria with asymmetric information, see Laffont [28]: he shows that partially revealing REE are not even ex-post Pareto optimal; in order to attain ex-post Pareto efficiency, full revelation is needed.
} 


\subsection{Overview}

The structure of the paper is as follows. In section 2 we present the model; sub-sections 2.1 and 2.2 characterise respectively the beliefs of informed and uninformed traders; finally sub-section 2.3 proves existence and shows conditions for uniqueness of a partially revealing: informational equilibrium.

Section 3 contains the main dynamic results of our study. In particular, sub-section 3.2 shows conditions for dominance of uninformed traders; sub-section 3.3 proves that, as long as information acquisition is costly, uninformed traders survive; in sub-section 3.4 we ask what is the long-run market outcome when the cost of information decreases and eventually goes to zero.

In section 4 we look at the population dynamics in a model where prices fully reveal the information of informed traders to the uninformed.

Section 5 concludes the paper.

For ease of exposition, proofs are in the appendix.

\section{The Model}

We consider a very stylised model of a financial market. Time is discrete and indexed by $t=0,1, \ldots$. There are $S$ payoff relevant states of the world and $S$ elementary assets: with little abuse of notation, both states of the world and assets are indexed by $s=1,2, \ldots, S$. At the end of each period, there is a random draw over states of the world. If state $s$ occurs, asset $s$ pays a strictly positive payoff $w_{s}>0$ and all other assets $s^{\prime} \neq s$ pay zero.

Traders do not observe the realisation of the random draw over states of the world prior to their trading. However, they can observe ex-ante the realisation of another random variable, (possibly) correlated with the state of the world, that we shall therefore refer to as signal. We will assume that there are as many signals as states of the world ${ }^{6}$ and we will index signals by $z=1, \ldots, S$. The joint probability distribution over states of the world and signals is constant over time and is common knowledge among traders. Therefore the probabilistic structure of the asset payouts can be summarised by the following probability matrix:

\begin{tabular}{l|llllll|l} 
& 1 & 2 & $\cdots$ & $s$ & $\cdots$ & $S$ & \\
\hline 1 & $p_{11}$ & $p_{21}$ & $\cdots$ & $p_{s 1}$ & $\cdots$ & $p_{S 1}$ & $\theta_{1}$ \\
2 & $p_{12}$ & $p_{22}$ & $\cdots$ & $p_{s 2}$ & $\cdots$ & $p_{S 2}$ & $\theta_{2}$ \\
$\vdots$ & $\vdots$ & $\vdots$ & $\ddots$ & $\vdots$ & $\ddots$ & $\vdots$ & $\vdots$ \\
$z$ & $p_{1 z}$ & $p_{2 z}$ & $\cdots$ & $p_{s z}$ & $\cdots$ & $p_{S z}$ & $\theta_{z}$ \\
$\vdots$ & $\vdots$ & $\vdots$ & $\ddots$ & $\vdots$ & $\ddots$ & $\vdots$ & $\vdots$ \\
$S$ & $p_{1 S}$ & $p_{2 S}$ & $\cdots$ & $p_{s S}$ & $\cdots$ & $p_{S S}$ & $\theta_{S}$ \\
\hline & $p_{1}$ & $p_{2}$ & $\cdots$ & $p_{s}$ & $\cdots$ & $p_{S}$ & 1
\end{tabular}

\footnotetext{
${ }^{6}$ We need the number of signals to be greater or equal to the number of states of the world because we want to allow for fully revealing signals. On the other hand, having more signals than states of the world would make the system informationally redundant. For this reason we assume that there are as many signals as states of the world.
} 
where $p_{s z} \geq 0$ denotes the joint probability $\operatorname{Pr}\{s, z\} ;\left\{\theta_{z}\right\}_{z=1}^{S}$ and $\left\{p_{s}\right\}_{s=1}^{S}$ are the marginal probability distributions respectively over signals and states of the world. Clearly:

$$
\begin{gathered}
\sum_{s=1}^{S} p_{s z}=\theta_{z} \\
\sum_{z=1}^{S} p_{s z}=p_{s} \\
\sum_{s=1}^{S} \sum_{z=1}^{S} p_{s z}=\sum_{z=1}^{S} \theta_{z}=\sum_{s=1}^{S} p_{s}=1
\end{gathered}
$$

The supply of asset $s$ at date $t, x_{s t}$, is such that $0<x_{s t}<\infty$. We assume that $x_{s t}$ is the random draw at date $t$ of a continuous random variable $X_{s}$ distributed between 0 and $\infty$ according to a continuous density function $f_{X_{s}}\left(x_{s}\right)$. We also assume that $X_{1}, X_{2}, \ldots, X_{S}$ are i.i.d. so that we can write $f_{X_{s}}\left(x_{s}\right)=f_{X}\left(x_{s}\right), \forall s$ and we can denote the joint density as $f(x)=\prod_{s=1}^{S} f_{X}\left(x_{s}\right)$. If state $s$ occurs at $t$, then the total wealth in the economy at the end of period $t$ will be $w_{t}=x_{s t} w_{s}$.

We are now ready to summarise the probabilistic structure of this stylised financial market. There are three sources of uncertainty represented by three random variables: the $S$-dimensional random vector of asset supplies $x_{t}$, the signal $z$ that informed traders observe and the state $s$ of the world that determines which asset will pay. The random vector $x_{t}$ is independent from $z$ and $s$, whereas $z$ and $s$ are (possibly) dependent random variables. We can fully describe the state of the economy at date $t$ as $\omega_{t} \in\{1, \ldots, S\} \times\{1, \ldots, S\} \times \Re_{+}^{S}$ where $\omega_{t}=\left(s, z, x_{t}\right)$ where $s$ and $z$ are respectively the state of the world ${ }^{7}$ and the signal at date $t$.

Denote by $\Omega=\times_{t=0}^{\infty}\left\{s, z, x_{t}\right\}$ the set of sample paths, with representative element $\omega=$ $\left(\omega_{0}, \omega_{1}, \ldots, \omega_{t}, \ldots\right)$. Define $\Omega^{t}=\times_{\tau=0}^{t}\left\{s, z, x_{\tau}\right\}$ with typical element $\omega^{t}$ for each $t=0,1,2, \ldots$ Let $\Im$ denote the product- $\sigma$-field on $\Omega$; finally let $\Im_{t}$ denote the sub- $\sigma$-field $\sigma\left(\omega^{t}\right)$ of $\Im$, so that $\left\{\Im_{t} ; t \geq 0\right\}$ is a natural filtration.

There is a finite number of traders in this economy, indexed by $i \in\{1, \ldots, I\}$. All traders are risk averse and endowed with a logarithmic utility function, which they maximise given their beliefs and their budget constraints. At date $t$, trader $i$ invests his earnings from period $t-1, w_{t-1}^{i}$, in the $S$ available assets. Let $\rho_{s t}$ be the market price of (one unit of) asset $s$ at date $t$. Trader $i$ takes the price vector $\rho_{t}=\left(\rho_{1 t}, \ldots, \rho_{S t}\right)$ as given, and chooses a portfolio $\left\{q_{s t}^{i}\right\}_{s=1}^{S}$ where $q_{s t}^{i}$ denotes his demand of asset $s$ at date $t$. It is often convenient to express his portfolio choice as a vector of portfolio weights $\left\{\alpha_{s t}^{i}\right\}_{s=1}^{S}$, where $\alpha_{s t}^{i}$ denotes the percentage of wealth that trader $i$ invests in asset $s$. Clearly, by definition, the following relation holds:

$$
\alpha_{s t}^{i} \equiv \frac{q_{s t}^{i} \rho_{s t}}{w_{t-1}^{i}}
$$

\footnotetext{
${ }^{7}$ In what follows, we will refer to a state $s$ as state of the world and to the description of the state of the economy $\omega_{t}$ as state of nature. When the meaning of the word is clear from the context, we might refer to either of them as state.
} 
We will sometimes refer to the vector $\alpha_{t}^{i}$ as a portfolio rule.

At the end of period $t$, the realisation of the random draw over states of the world is observed by all agents and the total payout $w_{t}$ is distributed among traders proportionately to the quantity of the randomly drawn asset that each of them owns. For example, if state $s$ occurs, the investment income that trader $i$ will cash at date $t$ is equal to the share of asset $s$ that he holds times the total payout of asset $s$. Therefore:

$$
w_{t}^{i}=q_{s t}^{i} w_{s}=\frac{\alpha_{s t}^{i} w_{t-1}^{i}}{\rho_{s t}} w_{s}
$$

This amount also constitutes trader $i$ 's endowment ${ }^{8}$ of wealth available for investment in period $t+1$.

We consider two types of traders: informed traders (type $I$ ) and uninformed traders (type $U)$. Informed traders observe the realisation of the random draw over signals before making: their portfolio decisions; uninformed traders do not have access to that kind of information. As a counterpart of their informative privilege, informed traders pay a per period cost at the beginning of each time period ${ }^{9}$ : namely they devote a constant percentage $c$ of their portfolio, with $0<c<1$, to information acquisition. All other information is free and common knowledge among agents. In particular, uninformed traders observe the market clearing price vector $\rho_{t}=\left(\rho_{1 t}, \ldots, \rho_{S t}\right)$ and try to infer from it some information on the realisation of the signal.

The information structure of this economy can be formalised as follows: denote by $\Phi_{t}^{I}$ and $\Phi_{t}^{U}$ the information sets of informed and uninformed traders respectively; we assume that $\Phi_{t}^{I}=\left\{\rho_{t}, z\right\}$ and $\Phi_{t}^{U}=\left\{\rho_{t}\right\}$ where $z$ is the signal observed at date $t$. Both types of traders have access to current prices ${ }^{10}$; whereas only informed traders observe the current realisation of the signal ${ }^{11}$.

The time line in figure 1 might help to visualise the order in which action takes place in the market we are modelling. Note that we call period $t$ the time between date $t-1$ and date $t$.

Since all traders are logarithmic utility maximisers, they all bet their beliefs: they choose a portfolio composition where each asset $s$ is held in a percentage equal to the probability

\footnotetext{
${ }^{8}$ We are not modelling savings decisions here. However all our results would still hold if both types of traders had the same savings rate. See Blume and Easley [6].

${ }^{9}$ Traders do not choose strategically whether to purchase information or not. This is a competitive market and they take both prices and the informativeness of prices as given. All our results would still hold (and in fact hold a fortiori) if we allowed traders to revise their policy for information acquisition. Suppose, for example, that in every time period a small number of traders had the chance to revise their information policy and switch from informed to uninformed or vice versa, comparing their expected utilities under both regimes: the dynamics would work in the same direction as the endogenous wealth dynamics and simply reinforce it.

${ }^{10}$ We could also assume that both types of traders have access to past history: this would not change our analysis, since both signals and states of the world are independent over time.

${ }^{11}$ In Grossman and Stiglitz [21] the information sets of informed and uninformed traders also differ in that the former observe asset supplies and the latter don't. In our setting, we do not need to assume that informed traders observe asset supplies, since the portfolio that traders choose is independent from asset supplies. This is clearly due to the fact that we characterise utilities as logarithmic. However all our results would still hold if we had to assume, as in Grossman and Stiglitz, that informed traders do observe asset supplies.
} 


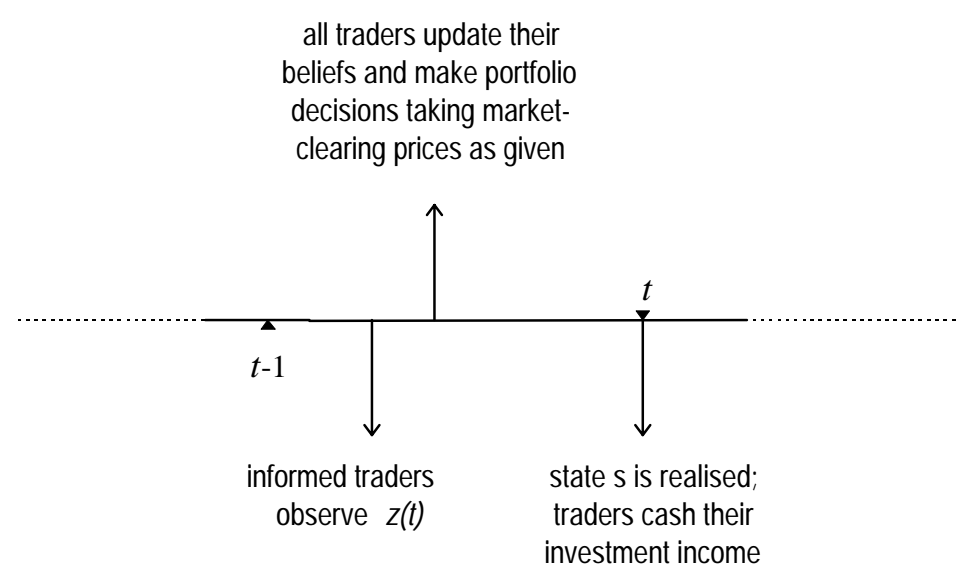

Figure 1: Time line.

that they attach to state $s$. The joint probability matrix is common knowledge among traders and we can interpret the marginal distributions $\left\{\theta_{z}\right\}_{z=1}^{S}$ and $\left\{p_{s}\right\}_{s=1}^{S}$ as their common priors respectively on the signal space and on the state space, which they update at each time period according to Bayes' rule. They use their posteriors to take portfolio decisions. We denote by $p_{t}^{I}=\left\{p_{s t}^{I}\right\}_{s=1}^{S}$ and $p_{t}^{U}=\left\{p_{s t}^{U}\right\}_{s=1}^{S}$ the posterior beliefs over the state space of informed and uninformed traders respectively.

We can formalise the portfolio choice of informed traders as follows:

$$
\begin{aligned}
& \max _{q_{t}^{I}} E\left[\log q_{s t}^{I} w_{s} \mid \Phi_{t}^{I}\right] \\
\text { s.t. } & \sum_{s=1}^{S} q_{s t}^{I} \rho_{s t}=(1-c) w_{t-1}^{I}
\end{aligned}
$$

The optimal portfolio for informed traders has weights:

$$
\alpha_{s t}^{I}=(1-c) p_{s t}^{I}
$$

and a portion $c$ of it will be devoted to information acquisition.

The portfolio choice of uninformed traders can be formalised as follows:

$$
\begin{array}{ll}
\max _{q_{t}^{U}} & E\left[\log q_{s t}^{U} w_{s} \mid \Phi_{t}^{U}\right] \\
\text { s.t. } & \sum_{s=1}^{S} q_{s t}^{U} \rho_{s t}=w_{t-1}^{U}
\end{array}
$$

and their optimal portfolio has weights:

$$
\alpha_{s t}^{U}=p_{s t}^{U}
$$




\subsection{The Beliefs of Informed Traders}

Informed traders observe the realisation of the signal ex ante: their posterior is equal to the probability distribution over the state space conditional on the signal they have observed. Suppose that signal $z$ is drawn and observed at $t$, then the posterior of the informed traders ${ }^{12}$, $p_{t}^{I}$, is as follows:

$$
p_{t}^{I}=\left\{\operatorname{Pr}\left\{s \mid \Phi_{t}^{I}\right\}=\operatorname{Pr}\{s \mid z\}=\frac{p_{s z}}{\theta_{z}}\right\}_{s=1}^{S}
$$

Clearly if signals and states of the world are independent, observing the realisation of the signal ex ante does not add any relevant information on the probability distribution over the state space. In fact independence implies, $\forall s, z \in\{1, \ldots, S\} \times\{1, \ldots, S\}$ :

$$
p_{s z}=p_{s} \theta_{z}
$$

which in turn implies:

$$
p_{t}^{I}=\left\{p_{s}\right\}_{s=1}^{S}
$$

namely the posterior of informed traders is equal to the common prior they started with. For signals to be informative we need some degree of dependence between signals and states.

As a measure of how informative the signal system is, we adopt the relative entropy measure between conditional and unconditional distributions.

Definition 1 (Relative entropy) Given two probability distributions $x=\left\{x_{s}\right\}_{s=1}^{S}$ and $y=$ $\left\{y_{s}\right\}_{s=1}^{S}$, the relative entropy of $y$ with respect to $x$ is

$$
\sum_{s=1}^{S} x_{s} \log \frac{x_{s}}{y_{s}}
$$

Although not a metric, the relative entropy measure provides us with a useful notion of distance between two probability distributions: it is always non-negative and it is equal to 0 if and only if $x_{s}=y_{s}, \forall s$.

We define as power of the signal $P$ the average relative entropy of unconditional with respect to conditional probability distributions.

\section{Definition 2 (Power of the signal)}

$$
P \equiv \sum_{z=1}^{S} \theta_{z} \sum_{s=1}^{S} \frac{p_{s z}}{\theta_{z}} \log \frac{p_{s z} / \theta_{z}}{p_{s}}
$$

\footnotetext{
${ }^{12}$ Notice that the information contained in market prices is not valuable to informed traders, as they already possess the superior information given by the realisation of the signal. Technically, the only information contained in prices is information about the signal that informed traders have observed, so that clearly: $\operatorname{Pr}\left\{s \mid \rho_{t}, z\right\}=\operatorname{Pr}\{s \mid z\}$.
} 
For example, suppose that, after observing signal $z$, the beliefs of the informed traders change considerably and become very "distant" from the initial prior. The "distance" between the prior and the posterior of the informed traders, is captured by the relative entropy of the probability distribution over the state space, conditional on signal $z$ being observed $\left\{p_{s z} / \theta_{z}\right\}_{s=1^{-}}^{S}$ with respect to the prior distribution $-\left\{p_{s}\right\}_{s=1}^{S}$ :

$$
\sum_{s=1}^{S} \frac{p_{s z}}{\theta_{z}} \log \frac{p_{s z} / \theta_{z}}{p_{s}}
$$

Averaging over $z$, we obtain a measure of how distant from the prior, the posterior of informed traders is on average. This can be easily interpreted as a measure of the power of the signal. In fact, if conditional probabilities are "close" to the prior, being able to observe the realisation of the signal ex-ante, is not very crucial. We will then say that the power of the signal is low. Conversely, if conditional probabilities are "far" from the prior, observing" the realisation of the signal ex-ante, will make a real difference. We will then say that the power of the signal is high.

Another useful notion is the notion of entropy, which can be interpreted as a measure of the unexpectedness of an event.

Definition 3 (Entropy) Given a probability distribution $x=\left\{x_{s}\right\}_{s=1}^{S}$, its entropy is

$$
E_{x}=-\sum_{s=1}^{S} x_{s} \log x_{s}
$$

The entropy of a probability distribution is a non-negative number which is equal to zero only when the probability distribution is degenerate and assigns probability 1 to one of the realisations and 0 to all the others, so that the unexpectedness of the random draw is null. On the other hand, the entropy reaches its global maximum when all probabilities are the same, so that the unexpectedness of the random draw is maximal.

Making use of the notion of entropy we can write the power of the signal as follows:

$$
P=E_{\theta}+E_{P R}-E_{p}
$$

where:

$$
\begin{aligned}
E_{\theta} & =-\sum_{z=1}^{S} \theta_{z} \log \theta_{z} \\
E_{P R} & =-\sum_{s=1}^{S} p_{s} \log p_{s} \\
E_{p} & =-\sum_{s=1}^{S} \sum_{z=1}^{S} p_{s z} \log p_{s z}
\end{aligned}
$$

and where $E_{\theta}$ represents the unexpectedness of the signal, $E_{P R}$ represents the unexpectedness of the state of the world (without knowing the signal realisation) and $E_{p}$ represents the 
unexpectedness of the state of the world once that a certain signal has occurred. The higher is the unexpectedness of the signal and the unexpectedness of the state of the world with respect to the unexpectedness of the joint random variable, the more valuable is the information added by observing the signal realisation.

In fact, when, for example, the unexpectedness of the signal is very low (hence $E_{\theta}$ is close to zero) then, even if uninformed traders cannot observe the signal realisation, their assessment on the random draw over signals is likely to be correct and therefore observing the signal is not very valuable. Similarly when the unexpectedness of the state of the world is not significantly reduced by observing the realisation of the signal (hence the difference $E_{P R}-E_{p} \leq 0$ is small), then again the signal is not very valuable.

As we noted above, when signals and states are independent signals are uninformative. In fact, if $\forall s, z$

$$
p_{s z}=p_{s} \theta_{z}
$$

then

$$
E_{\theta}+E_{P R}=E_{p} \Rightarrow P=0
$$

On the opposite side of the spectrum, we can get full information when signals and states are perfectly correlated. Consider the following probability matrix:

\begin{tabular}{c|cccc|l} 
& 1 & 2 & $\cdots$ & $S$ & \\
\hline 1 & $\theta_{1}$ & 0 & $\cdots$ & 0 & $\theta_{1}$ \\
2 & 0 & $\theta_{2}$ & $\cdots$ & 0 & $\theta_{2}$ \\
$\vdots$ & $\vdots$ & $\vdots$ & $\ddots$ & $\vdots$ & $\vdots$ \\
$S$ & 0 & 0 & $\cdots$ & $\theta_{S}$ & $\theta_{S}$ \\
\hline & $p_{1}=\theta_{1}$ & $p_{2}=\theta_{2}$ & $\cdots$ & $p_{S}=\theta_{S}$ & 1
\end{tabular}

In this case $E_{\theta}=E_{P R}=E_{p}$ and therefore ${ }^{13} P=E_{\theta}$. Namely the power of the signal lies in the unexpectedness of the value of $z$. In particular the informative privilege is the highest ${ }^{14}$ when the unexpectedness of the signal is the highest and therefore when $\theta_{z}=\theta=\frac{1}{S}, \forall z$. In this case the power of the signal $P$ reaches its upper bound $\log S$ so that we can claim that:

$$
0 \leq P \leq E_{\theta} \leq \log S
$$

In what follows, unless otherwise stated, we will assume signals to be informative but with some noise.

Assumption 1 (Noisy signals) The joint probability distribution over states of the world and signals is such that $\forall s, z \in\{1, \ldots, S\} \times\{1, \ldots, S\}$ :

$$
p_{s z}>0 \text { and } p_{s z} \neq p_{s} \theta_{z}
$$

And therefore $P$ is bounded away from zero and from $E_{\theta}$.

\footnotetext{
${ }^{13}$ Since $E_{P R}-E_{p} \leq 0$, then $P \leq E_{\theta}$ is always true.

${ }^{14}$ In this case, if the probability distribution over the signal space is degenerate so that one signal is assigned probability 1 and all other signals probability 0 , then $P=0$. In fact the informative privilege would disappear since there is no uncertainty over the signal space and therefore everyone virtually "observes" the signal.
} 


\subsection{The Beliefs of Uninformed Traders}

Uninformed traders do not observe the realisation of the signal. However they know the marginal probability distribution over the signal space and therefore attach to each signal realisation a prior belief. Moreover they can infer information from the market clearing price vector at $t, \rho_{t}$, and update their beliefs on which signal has been drawn, according to Bayes' rule.

Suppose, for instance, that signal $z$ places a high probability on state $s$. Informed traders, who can observe $z$, will choose a portfolio composition with a high weight on asset $s$. For this reason, when asset $s$ is traded at a high price, uninformed traders infer that state $s$ is more likely to occur as informed traders might have observed a "good" signal on asset s. As a result, they update their prior beliefs placing a higher probability on state $s$. The information than can be inferred from prices is therefore valuable. However it is noisy. In fact, when a trader observes a high price for asset $s$, he does not actually know if this is due to a "good" signal on $s$ being observed by informed traders or to a low supply of asset $s$.

If uninformed traders could observe the realisation of the random vector of asset supplies, prices would fully reveal which signals has been observed. However, we assume that this is not the case, so that the information contained in market prices is noisy.

According to Bayes' rule, the posterior of the uninformed traders over the signal space, $q_{t}$, is:

$$
q_{t}=\left\{q_{z t} \equiv \operatorname{Pr}\left\{z \mid \Phi_{t}^{U}\right\}=\operatorname{Pr}\left\{z \mid \rho_{t}\right\}=\frac{\operatorname{Pr}\left\{z \cap \rho_{t}\right\}}{\operatorname{Pr}\left\{\rho_{t}\right\}}\right\}_{z=1}^{S}
$$

and finally their posterior belief over the state space ${ }^{15} p_{t}^{U}$ is as follows:

$$
p_{t}^{U}=\left\{p_{s t}^{U} \equiv \operatorname{Pr}\left\{s \mid \rho_{t}\right\}=\sum_{z=1}^{S} q_{z t} \frac{p_{s z}}{\theta_{z}}\right\}
$$

Therefore in order to compute their posterior beliefs uninformed traders have to consider the probability distribution which is induced on market prices.

Fxistence of equilibrium will be discussed and proved in the next section. Let us assume, for the moment, that an equilibrium exists and let us characterise the beliefs of uninformed traders.

Denote by $\varepsilon_{t-1}^{I}=w_{t-1}^{I} / w_{t-1}$ the share of $w_{t-1}$ held by informed traders, so that $\left(1-\varepsilon_{t-1}^{I}\right)$ is the wealth share of uninformed traders available for investment at date $t$. The budget of uninformed traders for asset acquisition at date $t$ is therefore $\left(1-\varepsilon_{t-1}^{I}\right) w_{t-1}$. Because of the flow cost $c$ paid for information, the budget of informed traders for asset acquisition is $(1-c) \varepsilon_{t-1}^{I} w_{t-1}$.

\footnotetext{
${ }^{15}$ The reason we cannot compute directly a posterior over the state space such as:

$$
\operatorname{Pr}\left\{s \mid \rho_{t}\right\}=\frac{p_{s} \operatorname{Pr}\left\{\rho_{t} \mid s\right\}}{\sum_{s^{\prime}} p_{s^{\prime}} \operatorname{Pr}\left\{\rho_{t} \mid s^{\prime}\right\}}
$$

is that the price vector is determined ex ante and therefore prices do not depend on the realisation of the state. They vary, instead, according to the signal that informed traders observed.
} 
Aggregate demand for asset $s$ at date $t$ is:

$$
\left[(1-c) \varepsilon_{t-1}^{I} p_{s t}^{I}+\left(1-\varepsilon_{t-1}^{I}\right) p_{s t}^{U}\right] w_{t-1}
$$

Market clearing requires:

$$
x_{s t} \rho_{s t}=\left[(1-c) \varepsilon_{t-1}^{I} p_{s t}^{I}+\left(1-\varepsilon_{t-1}^{I}\right) p_{s t}^{U}\right] w_{t-1}
$$

so that equilibrium prices must satisfy:

$$
\rho_{s t}=\frac{(1-c) \varepsilon_{t-1}^{I} p_{s t}^{I}+\left(1-\varepsilon_{t-1}^{I}\right) p_{s t}^{U}}{x_{s t}} w_{t-1}
$$

If signal $z$ is drawn at time $t$ then the market clearing price of asset $s$ satisfies

$$
\rho_{s t}=\left[(1-c) \varepsilon_{t-1}^{I} \frac{p_{s z}}{\theta_{z}}+\left(1-\varepsilon_{t-1}^{I}\right) p_{s t}^{U}\right] \frac{w_{t-1}}{x_{s t}}
$$

Denote by $x_{t}^{z}=\left\{x_{s t}^{z}\right\}_{s=1}^{S}$ the vector of asset supplies such that:

$$
x_{s t}^{z}=\left[(1-c) \varepsilon_{t-1}^{I} \frac{p_{s z}}{\theta_{z}}+\left(1-\varepsilon_{t-1}^{I}\right) p_{s t}^{U}\left(\rho_{t}\right)\right] \frac{w_{t-1}}{\rho_{s t}}
$$

Observing the vector of market prices $\rho_{t}$, uninformed traders know that one of the following $S$ mutually exclusive events must have occurred: either $\left(z=1 ; x_{t}=x_{t}^{1}\right)$, or $\left(z=2 ; x_{t}=x_{t}^{2}\right)$, $\ldots$, or finally $\left(z=S ; x_{t}=x_{t}^{S}\right)$, so that the following two events are equivalent:

$$
\left\{\rho_{t}\right\}=\left\{\cup_{z=1}^{S}\left\{z \cap x_{t}^{z}\right\}\right\}
$$

Bayes' rule implies that:

$$
\operatorname{Pr}\left\{z \mid \rho_{t}\right\}=\frac{\operatorname{Pr}\left\{z \cap \rho_{t}\right\}}{\operatorname{Pr}\left\{\rho_{t}\right\}}=\frac{\operatorname{Pr}\left\{z \cap x_{t}^{z}\right\}}{\sum_{z^{\prime}=1}^{S} \operatorname{Pr}\left\{z^{\prime} \cap x_{t}^{z^{\prime}}\right\}}
$$

and finally, recalling that $z$ and $x_{t}$ are independent random variables:

$$
q_{z t}=\operatorname{Pr}\left\{z \mid \rho_{t}\right\}=\frac{\theta_{z} f\left(x_{t}^{z}\right)}{\sum_{z^{\prime}=1}^{S} \theta_{z^{\prime}} f\left(x_{t}^{z^{\prime}}\right)}
$$

where

$$
f\left(x_{t}^{z}\right)=\prod_{s=1}^{S} f_{X}\left(x_{s t}^{z}\right)
$$

As a result, the posterior of uninformed traders is:

$$
p_{s t}^{U}=\sum_{z=1}^{S} \frac{p_{s z}}{\theta_{z}} q_{z t}=\sum_{z=1}^{S} \frac{p_{s z}}{\theta_{z}} \cdot \frac{\theta_{z} f\left(x_{t}^{z}\right)}{\sum_{z^{\prime}=1}^{S} \theta_{z^{\prime}} f\left(x_{t}^{z^{\prime}}\right)}
$$

Notice that the vector $x_{t}^{z}$ is a function of the posterior itself, so that this is not an explicit solution for $p_{s t}^{U}$. We solve for the equilibrium in section 2.3 . 


\subsection{Informational Equilibrium}

In this setting, information acquisition and equilibrium prices have to be determined simultaneously. In fact, asset demands, and hence asset prices, depend on the information that traders acquire, and the information that (some) traders acquire depends on asset prices. In the framework we modeled, prices actually serve two functions: they clear markets and convey information. Existence of equilibrium is often problematic in general equilibrium models with asymmetric information, precisely for the double role played by market prices. As Green [19] pointed out, this "seems to be due to the fact that the space of prices in not large enough [...] to transmit all of the relevant data" [[19], p. 451].

Definition 4 (Informational Equilibrium) An informational equilibrium at date $t$ for this economy is an array of beliefs, portfolios and asset prices such that beliefs are computed according to Bayes' rule, given all available information; portfolios maximise expected utility, given traders' beliefs, and markets clear. Formally, an informational equilibrium is $\left\{\left(p_{s t}^{I}, p_{s t}^{U}\right),\left(q_{s t}^{I}, q_{s t}^{U}\right), \rho_{s t}\right\}_{s=1}^{S}$ such that, $\forall s \in\{1, \ldots, S\}$ :

- $p_{s t}^{I}$ satisfies 1 ;

- $p_{s t}^{U}$ satisfies 2 ;

- $q_{s t}^{I}=\arg \max E\left[\log w_{t}^{I} \mid\left\{p_{s t}^{I}\right\}_{s=1}^{S}\right]$;

- $q_{s t}^{U}=\arg \max E\left[\log w_{t}^{U} \mid\left\{p_{s t}^{U}\right\}_{s=1}^{S}\right]$;

- $q_{s t}^{I}+q_{s t}^{U}=x_{s t}$.

Recall that the equilibrium price of asset $s$ at date $t$ must satisfy:

$$
\rho_{s t}=\left[(1-c) \varepsilon_{t-1} \frac{p_{s z}}{\theta_{z}}+\left(1-\varepsilon_{t-1}\right) \sum_{z^{\prime}} \frac{p_{s z^{\prime}}}{\theta_{z^{\prime}}} \cdot \frac{\theta_{z^{\prime}} f\left(x_{t}^{z^{\prime}}\right)}{\sum_{z^{\prime \prime}} \theta_{z^{\prime \prime}} f\left(x_{t}^{z^{\prime \prime}}\right)}\right] \frac{w_{t-1}}{x_{s t}}
$$

where

$$
f\left(x_{t}^{z}\right)=f\left(x_{1 t}^{z}, \ldots, x_{s t}^{z}, \ldots, x_{S t}^{z}\right)=\prod_{s=1}^{S} f_{X}\left(x_{s t}^{z}\right)
$$

and

$$
x_{s t}^{z}\left(\rho_{t}\right)=\left[(1-c) \varepsilon_{t-1} \frac{p_{s z}}{\theta_{z}}+\left(1-\varepsilon_{t-1}\right) p_{s t}^{U}\left(\rho_{t}\right)\right] \frac{w_{t-1}}{\rho_{s t}}
$$

The only condition we need in order to guarantee existence of equilibrium is continuity of the density function $f_{X}\left(x_{s}\right)$.

Proposition 5 (Existence) If $f_{X}\left(x_{s}\right)$ is continuous, then an informational equilibrium always exists in this economy. 
Proof. See appendix.

The proof of proposition 5 is straightforward. We first postulate that the beliefs of uninformed traders are a continuous function of equilibrium asset prices. We then prove that an equilibrium exists under this assumption and we check that the posterior of uninformed traders is a continuous function of asset prices. As a result, an equilibrium with beliefs that are continuous in prices will always exist. This does not guarantee, however, uniqueness.

In order to be able to analyse the dynamic properties of this model, we would like the equilibrium to be unique. For this reason, our next question is whether we can characterise a set of density functions such that equilibrium is also unique.

Recall that the posterior of uninformed traders maps a vector of prices into the $S$ dimensional unit simplex ${ }^{16}$ :

$$
p_{t}^{U}: \mathbb{R}_{+}^{S} \rightarrow \Delta^{S}
$$

Call $\mathcal{P}_{t}$ the set of all such mappings. Given a $p_{t}^{U} \in \mathcal{P}_{t}$, the random vector of supplies, $x_{t}$, induces a joint probability distribution on $\left(\rho_{t}, z\right)$, through market clearing:

$$
\rho_{s t}=\left[(1-c) \varepsilon_{t-1} \frac{p_{s z}}{\theta_{z}}+\left(1-\varepsilon_{t-1}\right) p_{s t}^{U}\left(\rho_{t}\right)\right] \frac{w_{t-1}}{x_{s t}}
$$

Call $\mathcal{H}_{t}$ the set of all the probability distributions over $\left(\rho_{t}, z\right)$; market clearing can therefore be seen as a mapping from $\mathcal{P}_{t}$ to $\mathcal{H}_{t}$ :

$$
\text { market clearing : } \mathcal{P}_{t} \rightarrow \mathcal{H}_{t}
$$

On the other hand, given a probability distribution over $\left(\rho_{t}, z\right)$, Bayes' rule gives us a posterior over states of the world:

$$
\text { Bayes' rule }: \mathcal{H}_{t} \rightarrow \mathcal{P}_{t}
$$

Equilibria, as a result, are a fixed point of the mapping:

$$
\varphi_{t}: \mathcal{P}_{t} \rightarrow \mathcal{P}_{t}
$$

We can make the functional space $\mathcal{P}_{t}$ into a complete metric space associating with each $p_{t}^{U} \in \mathcal{P}_{t}$ its supremum norm. $\mathcal{P}_{t}$ is the set of all continuous functions with domain $\mathbb{R}_{+}^{S}$ and codomain in the $S$-dimensional unit simplex. We associate with each $p_{t}^{U} \in \mathcal{P}_{t}$ its supremum norm:

$$
\left\|p_{t}^{U}\right\|=\sup _{s, \rho_{t}}\left|p_{t}^{U}\left(\rho_{t}\right)\right|
$$

We define the distance between $p_{t}^{U} \in \mathcal{P}_{t}$ and $g_{t}^{U} \in \mathcal{P}_{t}$ to be:

$$
\left\|p_{t}^{U}-g_{t}^{U}\right\|=\sup _{s, \rho_{t}}\left|p_{t}^{U}\left(\rho_{t}\right)-g_{t}^{U}\left(\rho_{t}\right)\right|
$$

and we have thus made $\mathcal{P}_{t}$ into a metric space which can be proved to be complete ${ }^{17}$.

\footnotetext{
${ }^{16}$ Notice that we do not normalise asset prices, so that the price space is not the simplex, but $\mathbb{R}_{+}^{S}$.

${ }^{17}$ See, for example, Rudin [32], p.151.
} 
Since $\mathcal{P}_{t}$ is a complete metric space, if the mapping $\varphi_{t}: \mathcal{P}_{t} \rightarrow \mathcal{P}_{t}$ is a contraction, then there exists one and only one fixed point. Namely, if $\exists \nu<1$ such that, $\forall p_{t}^{U}, g_{t}^{U} \in \mathcal{P}_{t}$

$$
\left\|\varphi_{t}\left(p_{t}^{U}\right)-\varphi_{t}\left(g_{t}^{U}\right)\right\| \leq \nu\left\|p_{t}^{U}-g_{t}^{U}\right\|
$$

then $\varphi_{t}\left(p_{t}^{U}\right)$ is a contraction and equilibrium is unique.

We can show that a sufficient condition so that the posterior of uninformed traders is a contraction mapping is that prices are sufficiently distant from fully revealing. Namely, we need likelihood ratios to be bounded. Denote by $r_{t}\left(z, z^{\prime}, p_{t}^{U}\right)$ the likelihood ratio at date $t$ of signal $z^{\prime}$ with respect to $z$ when the posterior function is $p_{t}^{U}$ :

$$
r_{t}\left(z, z^{\prime}, p_{t}^{U}\right)=\frac{f\left(x_{t}^{z^{\prime}}\left(p_{t}^{U}\right)\right)}{f\left(x_{t}^{z}\left(p_{t}^{U}\right)\right)}
$$

For full revelation to occur at date $t$, we need that, $\forall z^{\prime} \in\{1, \ldots, S\}, r_{t}\left(z, z^{\prime}, p_{t}^{U}\right)=0$ when $z$ is the true signal being observed and $r_{t}\left(z, z^{\prime}, p_{t}^{U}\right)=\infty$ when the true signal being observed is other than $z$. Bounded likelihood ratios imply only partial revelation.

We can finally claim the following:

Proposition 6 (Uniqueness) If $\forall z, z^{\prime}$ and $\forall p_{t}^{U}, g_{t}^{U} \in \mathcal{P}_{t}, \exists \nu<1$ such that:

$$
\frac{\left\|r_{t}\left(z, z^{\prime}, p_{t}^{U}\right)-r_{t}\left(z, z^{\prime}, g_{t}^{U}\right)\right\|}{\left\|p_{t}^{U}-g_{t}^{U}\right\|} \leq \nu \theta_{z}
$$

then equilibrium is unique.

Proof. See appendix.

Remark 1 If, $\forall z \in\{1, \ldots, S\}$, likelihood ratios lie in a range whose size is bounded away from $\theta_{z}$ then the condition in proposition 6 is satisfied.

In fact, suppose that $\exists a_{z}, b_{z}$ such that, $\forall z^{\prime}, p_{t}^{U}$ :

$$
a_{z} \leq r_{t}\left(z, z^{\prime}, p_{t}^{U}\right) \leq b_{z}
$$

then clearly:

$$
\left\|r_{t}\left(z, z^{\prime}, p_{t}^{U}\right)-r_{t}\left(z, z^{\prime}, g_{t}^{U}\right)\right\| \leq b_{z}-a_{z}
$$

so that the condition in proposition 6 is certainly satisfied if:

$$
b_{z}-a_{z} \leq \nu \theta_{z}\left\|p_{t}^{U}-g_{t}^{U}\right\|
$$

As we argued above, imposing a bounded range on likelihood ratios corresponds to requiring that prices have to be sufficiently far from full revelation.

A family of density functions that clearly satisfies the condition in proposition 6 is the exponential. In fact, when $f\left(x_{t}\right)$ is exponential, $\forall p_{t}^{U}, g_{t}^{U} \in \mathcal{P}_{t}$ :

$$
r_{t}\left(z, z^{\prime}, p_{t}^{U}\right)-r_{t}\left(z, z^{\prime}, g_{t}^{U}\right)=0
$$


Example 7 (Exponential density function) Assume that, $\forall s \in\{1, \ldots, S\}, X_{s}$ are exponentially distributed with mean ${ }^{18}$ equal to 1 . The joint density of asset supplies will be:

$$
f\left(x_{t}\right)=e^{-\sum_{s} x_{s t}}
$$

We can solve for the posterior of uninformed traders and get:

$$
p_{s t}^{U}=\sum_{z} \frac{p_{s z}}{\sum_{z^{\prime}} \theta_{z^{\prime}} \exp \left[-(1-c) \varepsilon_{t-1} w_{t-1} \sum_{s^{\prime}}\left(p_{s^{\prime} z^{\prime}} / \theta_{z^{\prime}}-p_{s^{\prime} z} / \theta_{z}\right) / \rho_{s^{\prime}}\right]}
$$

In what follows we will assume that the density function $f\left(x_{t}\right)$ satisfies condition 4 in proposition 6 , so that equilibrium is unique.

It is interesting to characterise the limiting beliefs of uninformed traders when the market is almost entirely populated by only one type of traders. We might expect that the quality of information that can be revealed through market prices is a function of the number of traders who are informed in the economy, so that if more people are informed, prices reflect more information.

Consider the limit of $p_{s t}^{U}$ for $\varepsilon_{t}^{I} \rightarrow 0$, namely the limiting posterior of uninformed traders when almost everyone in the economy is uninformed. In this case $x_{s t}^{z} \rightarrow p_{s t}^{U} w_{t-1} / \rho_{s t}$ and therefore its limiting value does not depend on $z$, so that:

$$
\lim _{\varepsilon_{t}^{I} \rightarrow \mathbf{0}} p_{s t}^{U}=\sum_{z=1}^{S} \frac{p_{s z}}{\theta_{z}} \cdot \frac{\theta_{z} f\left(x_{t}^{z}\right)}{\sum_{z^{\prime}} \theta_{z^{\prime}} f\left(x_{t}^{z^{\prime}}\right)}=\sum_{z=1}^{S} p_{s z}=p_{s}
$$

As we expected, we find that when almost everybody is uninformed, market prices contain virtually no information and the posterior of uninformed traders is equal to their prior.

At the other end of the spectrum, when $\varepsilon_{t}^{I} \rightarrow 1$, we find that

$$
x_{s t}^{z} \rightarrow \frac{(1-c) p_{s z} / \theta_{z}}{\rho_{s t}} w_{t-1}
$$

so that $f\left(x_{t}^{z^{\prime}}\right) / f\left(x_{t}^{z^{\prime}}\right) \neq 1$ iff $z^{\prime} \neq z$ and the posterior of uninformed traders is different from their prior, so that they indeed succeed in extracting some information from market prices. We ask now whether the information inferred from market prices is complete, so that equilibrium prices are fully revealing.

Definition 8 (Full revelation) An informational equilibrium is fully revealing if the posteriors of informed and uninformed traders agree, so that:

$$
\forall t \geq 0, \quad p_{t}^{I}=p_{t}^{U}
$$

It is straightforward to show that, as long as there is noise in the system, uninformed traders cannot fully extract the information of informed traders from market prices.

Corollary 9 (Non-Fully Revealing Equilibrium) Under assumption 1, the informational equilibrium in this economy is not fully revealing.

Proof. See appendix.

\footnotetext{
${ }^{18}$ Taking the parameter of the exponential equal to 1 is for simplicity only.
} 


\section{The Evolution of Wealth Shares}

In this paper we are interested in assessing the evolutionary fitness of the two types of traders we described in the previous section. We shall look at the evolution of their wealth shares to characterise what happens in the long run. Let us describe the wealth dynamics of informed traders first. If signal $z$ has been drawn and if state $s$ occurred at $t$, then the investment income of an informed trader, $w_{t}^{I}$, will be equal to the share of asset $s$ that he holds times the total payout of asset $s$. Therefore:

$$
w_{t}^{I}=\underbrace{\frac{(1-c) w_{t-1}^{I} p_{s z} / \theta_{z}}{x_{s t} \rho_{s t}}}_{\begin{array}{c}
\text { share of asset } s \text { that } \\
\text { informed traders hold }
\end{array}} \underbrace{x_{s t} w_{s}}_{\begin{array}{c}
\text { total payout } \\
\text { at date } t, w_{t}
\end{array}}
$$

Denote by $\varepsilon_{t}^{I}=w_{t}^{I} / w_{t}$ the wealth share of informed traders, so that we can write:

$$
\varepsilon_{t}^{I}=\frac{(1-c) p_{s z} / \theta_{z}}{x_{s t} \rho_{s t} / w_{t-1}} \varepsilon_{t-1}^{I}
$$

Equation 5 fully describes the period to period dynamics of the wealth share of informed traders if $z$ and $s$ occur at $t$.

Similarly we can describe the dynamics of the wealth share of uninformed traders. Denote by $w_{t}^{U}$ the investment income of uninformed traders at $t$ if state $s$ occurs. Clearly:

$$
w_{t}^{U}=\underbrace{\underbrace{\frac{w_{t-1}^{U} p_{s t}^{U}}{x_{s t} \rho_{s t}}}_{\text {total payout }}}_{\begin{array}{c}
\text { share of asset } s \text { that } \\
\text { uninformed traders hold }
\end{array}} \underbrace{x_{s t} w_{s}}_{\text {at date } t, w_{t}}
$$

Denote by $\varepsilon_{t}^{U}=w_{t}^{U} / w_{t}$ and get:

$$
\varepsilon_{t}^{U}=\frac{p_{s t}^{U}}{x_{s t} \rho_{s t} / w_{t-1}} \varepsilon_{t-1}^{U}
$$

Equations 5 and 6 represent a fitness-monotonic dynamic: the wealth share of informed (uninformed) traders will increase if and only if they score a payoff which is higher than the average population payoff. In fact, the ratio which appears in the denominator of 5 and 6 can be rewritten as follows:

$$
\frac{x_{s t} \rho_{s t}}{w_{t-1}}=(1-c) \frac{p_{s z}}{\theta_{z}} \varepsilon_{t-1}^{I}+p_{s t}^{U}\left(1-\varepsilon_{t-1}^{I}\right)
$$

and expression 7 clearly gives us a measure of the average population payoff.

We are interested in characterising the limiting behaviour of wealth shares as time goes to infinity and in determining under which conditions each type of traders dominates, survives or vanishes according to the following definition borrowed by Blume and Easley [6]: 
Definition 10 (a) Trader $i$ dominates on the set of sample paths $Q \in \Im$ if :

$$
\lim _{t \rightarrow \infty} \inf \varepsilon_{t}^{i}>0 \text { a.s. on } Q
$$

(b) Trader $i$ survives on the set of sample paths $Q \in \Im$ if :

$$
\lim _{t \rightarrow \infty} \sup \varepsilon_{t}^{i}>0 \quad \text { a.s. on } Q \text {; }
$$

(c) Trader $i$ vanishes on the set of sample paths $Q \in \Im$ if :

$$
\lim _{t \rightarrow \infty} \sup \varepsilon_{t}^{i}=0 \quad \text { a.s. on } Q \text {. }
$$

According to definition 10, in order to establish whether informed (or uninformed) traders dominate on a sample path $\omega=\left(\omega_{1}, \omega_{2}, \ldots, \omega_{t}, \ldots\right)$ we need to consider the asymptotic behaviour of their wealth share: namely we have to check whether it is bounded away from zero or not. Note that the wealth share of a dominating trader does not necessarily converge to 1 , as there might be other surviving traders.

Moreover, market dominance is clearly related to the process of price determination: differential rates of wealth accumulation increase the relative significance of some individuals in determining market outcomes and namely equilibrium prices. In particular, Blume and Easley [6] justify their use of the word "dominance" as follows: "when savings rates are identical, a trader who dominates actually determines the price asymptotically. His wealth share need not converge to one because there may be other traders who asymptotically have the same portfolio rule, but prices adjust so that his conditional expected gains converge to zero" ([6], p. 19). This consideration also justifies the distinction between dominance and mere survival.

In order to study the asymptotic properties of wealth shares we can rely on some intermediate results.

First, we show that $\log \varepsilon_{t}^{i}$ is the sum of uniformly bounded dependent random variables. Because of dependence we cannot apply the law of large numbers; however, thanks to uniform boundedness, we can use results known to apply to sums of uniformly bounded random variables and which do not require independence.

Consider the wealth share of informed traders. Given a sample path, we can fully describe the dynamics of wealth shares and recursively solve equation 5 so that it is possible to express $\varepsilon_{t}^{I}$ as a function of the initial wealth share $\varepsilon_{0}^{I}$ :

$$
\begin{aligned}
\varepsilon_{t}^{I} & =\prod_{\tau=1}^{t} \prod_{s=1}^{S} \prod_{z=1}^{S} \iota_{s z \tau} \frac{(1-c) p_{s z} / \theta_{z}}{x_{s \tau} \rho_{s \tau} / w_{\tau-1}} \varepsilon_{0}^{I}= \\
& =\prod_{\tau=1}^{t} \prod_{s=1}^{S} \prod_{z=1}^{S} \iota_{s z \tau} \frac{(1-c) p_{s z} / \theta_{z}}{\varepsilon_{\tau-1}^{I}(1-c) p_{s z} / \theta_{z}+\left(1-\varepsilon_{\tau-1}^{I}\right) \sum_{z^{\prime}} q_{z^{\prime} \tau}\left(p_{s z^{\prime}} / \theta_{z^{\prime}}\right)} \varepsilon_{0}^{I}
\end{aligned}
$$

where $\iota_{s z t}$ is an indicator function that is equal to 1 if $(s, z)$ at $t$ and to 0 otherwise. We can take logs of equation 8 and express it as a sum of random variables:

$$
\log \varepsilon_{t}^{I}=\sum_{\tau=1}^{t} \sum_{s=1}^{S} \sum_{z=1}^{S} \iota_{s z \tau} \log \frac{(1-c) p_{s z} / \theta_{z}}{\varepsilon_{\tau-1}^{I}(1-c) p_{s z} / \theta_{z}+\left(1-\varepsilon_{\tau-1}^{I}\right) \sum_{z^{\prime}} q_{z^{\prime} \tau}\left(p_{s z^{\prime}} / \theta_{z^{\prime}}\right)}+\log \varepsilon_{\mathbf{0}}^{I}
$$


Let:

$$
\sum_{s=1}^{S} \sum_{z=1}^{S} \iota_{s z t} \log \frac{(1-c) p_{s z} / \theta_{z}}{\varepsilon_{t-1}^{I}(1-c) p_{s z} / \theta_{z}+\left(1-\varepsilon_{t-1}^{I}\right) \sum_{z^{\prime}} q_{z^{\prime} t}\left(p_{s z^{\prime}} / \theta_{z^{\prime}}\right)}=\lambda_{t}^{I}
$$

and finally:

$$
\log \varepsilon_{t}^{I}=\sum_{\tau=1}^{t} \lambda_{\tau}^{I}+\log \varepsilon_{0}^{I}
$$

Clearly $\lambda_{t}^{I}$ depends on past wealth shares and, through asset prices, on the past level of wealth. As a result, $\lambda_{t}^{I}$ are not independent random variables.

We can show, however, that the random variables $\lambda_{t}^{I}$ are uniformly bounded. In fact, $\forall t \geq 0$ :

$$
\log \frac{(1-c) \min _{s, z}\left\{p_{s z} / \theta_{z}\right\}}{\max _{s, z}\left\{p_{s z} / \theta_{z}\right\}} \leq \lambda_{t}^{I} \leq \log \frac{(1-c) \max _{s, z}\left\{p_{s z} / \theta_{z}\right\}}{\min _{s, z}\left\{p_{s z} / \theta_{z}\right\}}
$$

Similarly, looking at the wealth share of uninformed traders, we can show that:

$$
\log \varepsilon_{t}^{U}=\sum_{\tau=1}^{t} \lambda_{\tau}^{U}+\log \varepsilon_{0}^{U}
$$

where, $\forall t \geq 0$ :

$$
\log \frac{\min _{s, z}\left\{p_{s z} / \theta_{z}\right\}}{\max _{s, z}\left\{p_{s z} / \theta_{z}\right\}} \leq \lambda_{t}^{U} \leq \log \frac{\max _{s, z}\left\{p_{s z} / \theta_{z}\right\}}{\min _{s, z}\left\{p_{s z} / \theta_{z}\right\}}
$$

Uniform boundedness is crucial, since it allows us to state the following intermediate result:

Lemma 11 Trader $i$ dominates on the set of sample paths $Q \in \Im$ if:

$$
\lim _{t \rightarrow \infty} \inf \sum_{\tau=0}^{t} E\left[\lambda_{\tau}^{i} \mid \Im_{\tau-1}\right]>-\infty \text { a.s. on } Q
$$

Proof. See appendix.

The proof of lemma 11 relies on a theorem that essentially claims that the limiting behaviour of sums of uniformly bounded random variables cannot be different from the limiting behaviour of the sum of their conditional means. Intuitively, this occurs because uniformly bounded random variables cannot display large deviations from their expected values. The formal proof builds on corollary 4.5 in Freedman [16] and on lemmas A.1 and A.2 in Blume and Easley [6].

Lemma 11 is crucial since it allows us to work directly on expected values rather than on random variables. It claims, in fact, that, provided that $\lambda_{t}^{i}$ are uniformly bounded random variables, $\liminf \operatorname{in}_{t \rightarrow \infty} \sum_{\tau=0}^{t} E\left[\lambda_{\tau}^{i} \mid \Im_{\tau-1}\right]>-\infty$ almost surely, implies $\liminf _{t \rightarrow \infty} \sum_{\tau=0}^{t} \lambda_{\tau}^{i}>$ $-\infty$ almost surely. 


\subsection{The Evolutionary Advantage and Disadvantage of Costly In- formation}

Costly information triggers a trade-off in our setting. Informed traders enjoy an informative advantage with respect to uninformed traders and intuition suggests that this advantage is higher when most of the agents are uninformed. On the other hand informed traders have to devote a percentage of their wealth to information acquisition and bear a cost disadvantage with respect to uninformed traders. This disadvantage will be higher when most of the agents are informed. If a trader could choose whether to purchase information or not, he would have to face the trade-off between advantage and disadvantage of being informed and he would have to make his choice accordingly. Since both the advantage and disadvantage of costly information depend on the market share of informed agents with respect to uninformed ones, we can conjecture that there will be a population composition between informed and uninformed traders such that a rational trader would be indifferent between acquiring information or not. Such population shares stand as a strong candidate for a stable evolutionary equilibrium in our setting.

The trade-off we have just described becomes clear when we formalise it. Recall the period to period dynamics of the wealth share of informed traders:

$$
\varepsilon_{t}^{I}=\frac{(1-c) p_{s z} / \theta_{z}}{x_{s t} \rho_{s t} / w_{t-1}} \varepsilon_{t-1}^{I}
$$

The aggregate budget constraint requires that the aggregate expenditure in assets must be equal to the aggregate wealth invested in assets:

$$
\sum_{s=1}^{S} x_{s t} \rho_{s t}=\left[(1-c) \varepsilon_{t-1}^{I}+\left(1-\varepsilon_{t-1}^{I}\right)\right] w_{t-1}=\left(1-c \varepsilon_{t-1}^{I}\right) w_{t-1}
$$

so that we can rewrite equation 9 as follows:

$$
\varepsilon_{t}^{I}=\frac{(1-c) p_{s z} / \theta_{z}}{x_{s t} \rho_{s t}} w_{t-1} \frac{\sum_{s=1}^{S} x_{s t} \rho_{s t}}{\left(1-c \varepsilon_{t-1}^{I}\right) w_{t-1}} \varepsilon_{t-1}^{I}
$$

and finally:

$$
\varepsilon_{t}^{I}=\frac{p_{s z} / \theta_{z}}{x_{s t} \rho_{s t} / \sum_{s=1}^{S} x_{s t} \rho_{s t}} \cdot \frac{1-c}{1-c \varepsilon_{t-1}^{I}} \cdot \varepsilon_{t-1}^{I}
$$

Expression 10 fully describes the dynamics of the wealth share of informed traders from date $t-1$ to date $t$, if $\left\{s, z, x_{t}\right\}$ at $t$. We can take logs of expression 10 and get that, if $\left\{s, z, x_{t}\right\}$ at $t$, then:

$$
\log \varepsilon_{t}^{I}=\log \frac{p_{s z} / \theta_{z}}{x_{s t} \rho_{s t} / \sum_{s=1}^{S} x_{s t} \rho_{s t}}+\log \frac{1-c}{1-c \varepsilon_{t-1}^{I}}+\log \varepsilon_{t-1}^{I}
$$

We can now take the expected value of 11 and get:

$$
E\left[\log \varepsilon_{t}^{I} \mid \Im_{t-1}\right]=
$$




$$
\begin{aligned}
& \int_{0}^{\infty} \sum_{z=1}^{S} \theta_{z} \sum_{s=1}^{S} \frac{p_{s z}}{\theta_{z}} \log \frac{p_{s z} / \theta_{z}}{x_{s t} \rho_{s t} / \sum_{s=1}^{S} x_{s t} \rho_{s t}} f\left(x_{t}\right) d x_{t}+ \\
& +\log \frac{1-c}{1-c \varepsilon_{t-1}^{I}}+\log \varepsilon_{t-1}^{I}
\end{aligned}
$$

where $d x_{t}$ stands for $\prod_{s} d x_{s t}$; and where clearly, by independence of $\{s, z\}$ from $x_{t}, \operatorname{Pr}\left[s, z, x_{t}\right]=$ $\operatorname{Pr}[s, z] \operatorname{Pr}\left[x_{t}\right]=p_{s z} f\left(x_{t}\right)$.

The first term of equation 12 is always non-negative and it is equal to zero if and only if prices are such that $x_{s t} \rho_{s t} / \sum_{s=1}^{S} x_{s t} \rho_{s t}=p_{s z} / \theta_{z}, \forall s, z$. In fact the expression:

$$
\sum_{s=1}^{S} \frac{p_{s z}}{\theta_{z}} \log \frac{p_{s z} / \theta_{z}}{x_{s t} \rho_{s t} / \sum_{s=1}^{S} x_{s t} \rho_{s t}} \geq 0
$$

is the relative entropy of $\left\{x_{s t} \rho_{s t} / \sum_{s=1}^{S} x_{s t} \rho_{s t}\right\}_{s=1}^{S}$ with respect to $\left\{p_{s z} / \theta_{z}\right\}_{s=1}^{S}$. The second term of equation 12 is always negative because $(1-c)<\left(1-c \varepsilon_{t-1}^{I}\right)$ as long as $\varepsilon_{t-1}^{I}<1$.

The positive component, therefore, represents the evolutionary advantage given by information. Informed traders are in fact adopting the fittest portfolio rule which is investing according to probabilities ${ }^{19}$. Their evolutionary advantage is the highest when the distance from probabilities (as measured by relative entropy) of the portfolio rule that the market on average is adopting, is the highest. As we suggested above, we can therefore expect their evolutionary advantage to be highest when their wealth share is close to zero, so that almost everyone is uninformed and the market is on average far from the fittest portfolio rule.

The negative component is the cost disadvantage imposed by costly information acquisition. The disadvantage in evolutionary terms is the lowest when the wealth share of informed traders is close to 1 .

In fact, suppose that almost everyone is purchasing information and paying the information cost $c$, then clearly the disadvantage of costly information acquisition relative to the rest of the population of traders will be very low. On the contrary, when there are only a few traders who bear the cost of information acquisition, clearly the disadvantage relative to the rest of the population of traders will be the highest.

In order to be more precise let us denote by:

$$
\begin{gathered}
A D V_{t}\left(\varepsilon_{t-1}^{I}\right)=\int_{0}^{\infty} \sum_{z=1}^{S} \theta_{z} \sum_{s=1}^{S} \frac{p_{s z}}{\theta_{z}} \log \frac{p_{s z} / \theta_{z}}{x_{s t} \rho_{s t} / \sum_{s=1}^{S} x_{s t} \rho_{s t}} f\left(x_{t}\right) d x_{t} \\
C_{t}\left(\varepsilon_{t-1}^{I}\right)=-\log \frac{1-c}{1-c \varepsilon_{t-1}^{I}}>0
\end{gathered}
$$

so that:

$$
E\left[\log \varepsilon_{t}^{I} \mid \Im_{t-1}\right]=A D V_{t}\left(\varepsilon_{t-1}^{I}\right)-C_{t}\left(\varepsilon_{t-1}^{I}\right)+\log \varepsilon_{t-1}^{I}
$$

Equation 13 is central to the functioning of our model and it fully describes the period to period average dynamics of the wealth share of informed traders. When the advantage of

\footnotetext{
${ }^{19}$ See Blume and Easley [6].
} 
being informed offsets the cost of information, then on average the wealth share of informed traders increases, as they do better than their uninformed counterpart. When, on the contrary, the advantage of being informed is offset by the cost of information, then uninformed traders do better and the wealth share of informed traders decreases on average.

In order to be able to characterise the long-run distribution of wealth between informed and uninformed traders, we need to study the behaviour of the advantage and disadvantage of information acquisition, as functions of traders' wealth shares. Intuition suggests that the advantage of being informed is a decreasing function of $\varepsilon_{t-1}^{I}$, while the cost of being informed is an increasing function of $\varepsilon_{t-1}^{I}$.

The second result is straightforward; simple calculus in fact proves the following:

Proposition 12 The cost of being informed $C_{t}\left(\varepsilon_{t-1}^{I}\right)$ is a monotonically increasing and concave function of $\varepsilon_{t-1}^{I}$.

Proof. Trivial.

We can characterise the value of the cost function at the two extremes:

$$
\begin{aligned}
& C_{t}\left(\varepsilon_{t-1}^{I}=1\right)=0 \\
& C_{t}\left(\varepsilon_{t-1}^{I}=0\right)=\log \frac{1}{1-c}
\end{aligned}
$$

The advantage of being informed as a function of $\varepsilon_{t-1}^{I}$, on the other hand, is a more complicated object. We can, however, characterise its value at the extremes and its upper bound, which can be shown to be monotonically decreasing.

We can in fact show that the advantage of being informed has an "interesting" upper bound in the evolutionary advantage that informed traders enjoy with respect to traders who do not infer information from market prices. In fact we can rewrite $A D V_{t}\left(\varepsilon_{t-1}^{I}\right)$ as follows:

$$
A D V_{t}\left(\varepsilon_{t-1}^{I}\right)=\int_{0}^{\infty} \sum_{z=1}^{S} \theta_{z} \sum_{s=1}^{S} \frac{p_{s z}}{\theta_{z}} \log \frac{p_{s z} / \theta_{z}}{\gamma_{t-1} p_{s z} / \theta_{z}+\left(1-\gamma_{t-1}\right) p_{s t}^{U}\left(\rho_{t}\right)} f\left(x_{t}\right) d x_{t}
$$

where

$$
\gamma_{t-1}=\frac{\varepsilon_{t-1}^{I}(1-c)}{\varepsilon_{t-1}^{I}(1-c)+\left(1-\varepsilon_{t-1}^{I}\right)}
$$

and claim the following:

Proposition 13 (Upper bound) The advantage of being informed $A D V_{t}\left(\varepsilon_{t-1}^{I}\right)$ is maximal when uninformed traders invest according to their priors:

$$
A D V_{t}\left(\varepsilon_{t-1}^{I}\right) \leq \sum_{s=1}^{S} \frac{p_{s z}}{\theta_{z}} \log \frac{p_{s z} / \theta_{z}}{\gamma_{t-1} p_{s z} / \theta_{z}+\left(1-\gamma_{t-1}\right) p_{s}}
$$


Proof. See appendix.

This result confirms the intuition that the information revealed by market prices does, in fact, reduce the evolutionary advantage of those traders who possess superior information.

Let us denote by $\overline{A D V}_{t}\left(\varepsilon_{t-1}^{I}\right)$ the upper bound of $A D V_{t}\left(\varepsilon_{t-1}^{I}\right)$, so that proposition 13 can be summarised as:

$$
\overline{A D V}_{t}\left(\varepsilon_{t-1}^{I}\right)=\sum_{s=1}^{S} \frac{p_{s z}}{\theta_{z}} \log \frac{p_{s z} / \theta_{z}}{\gamma_{t-1} p_{s z} / \theta_{z}+\left(1-\gamma_{t-1}\right) p_{s}}
$$

The upper bound of the evolutionary advantage of being informed is a much simpler function of $\varepsilon_{t-1}^{I}$, so that we can prove the following:

Proposition 14 The upper bound of the advantage of being informed, $\overline{A D V}_{t}\left(\varepsilon_{t-1}^{I}\right)$, is a monotonically decreasing function of the wealth share of the informed, $\varepsilon_{t-1}^{I}$.

Proof. See appendix.

Finally we can characterise the value of the advantage of being informed and of its upper bound at the two extremes for $\varepsilon_{t-1}^{I}$ :

$$
\begin{aligned}
& \overline{A D V}_{t}\left(\varepsilon_{t-1}^{I}=1\right)=A D V_{t}\left(\varepsilon_{t-1}^{I}=1\right)=0 \\
& \overline{A D V}_{t}\left(\varepsilon_{t-1}^{I}=0\right)=A D V_{t}\left(\varepsilon_{t-1}^{I}=0\right)=P>0
\end{aligned}
$$

where $P$ is the power of the signal and depends entirely on the exogenous parameters of the model.

Particularly interesting is the analysis of the behaviour of advantage and disadvantage of being informed in the proximity of $\varepsilon_{t-1}^{I}=1$. We can show that $A D V_{t}\left(\varepsilon_{t-1}^{I}\right)$ is flat at $\varepsilon_{t-1}^{I}=1$.

Proposition 15 The marginal advantage of being informed when almost everyone is informed is null. Formally:

$$
\lim _{\varepsilon_{t-1}^{I} \rightarrow 1} \frac{\partial A D V_{t}\left(\varepsilon_{t-1}^{I}\right)}{\partial \varepsilon_{t-1}^{I}}=0
$$

Proof. See appendix.

On the other hand it is immediate to show that the marginal disadvantage of being informed when almost everyone is informed is strictly negative as long as $c>0$ :

$$
\lim _{\varepsilon_{t-1}^{I} \rightarrow 1} \frac{\partial C_{t}\left(\varepsilon_{t-1}^{I}\right)}{\partial \varepsilon_{t-1}^{I}}=-\frac{c}{1-c}<0
$$

These preliminary results suggest that "everyone informed" is not a stable equilibrium. We can summarise our analysis in the diagram depicted in figure 2, where the solid lines represent the advantage and the disadvantage of being informed and the dotted line represents the upper bound for the evolutionary advantage ${ }^{20}$ of informed traders.

\footnotetext{
${ }^{20}$ Note that we are depicting the evolutionary advantage of being informed as a monotonically decreasing function of $\varepsilon_{t-1}^{I}$ for simplicity. We do not provide such a result analytically and do not, in fact, need it in what follows.
} 


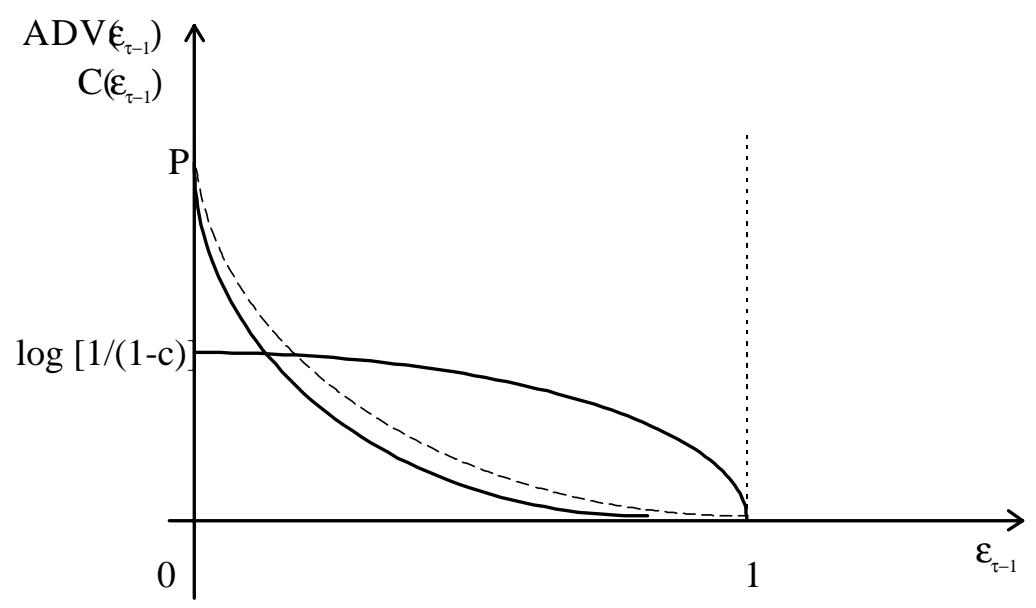

Figure 2: Advantage and disadvantage of being informed.

\subsection{Dominance of Uninformed Traders}

Suppose that the information available is not very good value for money. In particular, consider the case in which the flow cost that informed traders pay in order to purchase information is very high with respect to the quality of the information purchased, as measured by the power of the signal $P$. Namely suppose that the flow cost paid for information is above a threshold level given by $\left(e^{P}-1\right) / e^{P}$; namely ${ }^{21}$ :

$$
c \geq \frac{e^{P}-1}{e^{P}}
$$

so that:

$$
\log \frac{1}{1-c} \geq P
$$

then clearly, as depicted in figure 3, the cost function is above the upper bound of the evolutionary advantage function. A fortiori: $\forall t \geq 0: A D V_{t}\left(\varepsilon_{t-1}^{I}\right) \leq C_{t}\left(\varepsilon_{t-1}^{I}\right)$ and:

$$
E\left[\log \varepsilon_{t}^{I} \mid \Im_{t-1}\right] \leq \log \varepsilon_{t-1}^{I}
$$

so that $\log \varepsilon_{t}^{I}$ is a process that decreases on average, a supermartingale.

In this case we can show that uninformed traders dominate and drive to extinction traders who purchase the available information.

Proposition 16 (Dominance of Uninformed Traders) If $c$ is such that

$$
\frac{e^{P}-1}{e^{P}} \leq c \leq 1
$$

\footnotetext{
${ }^{21}$ Notice that the exponential term appears in the threshold level because the actual "utility cost" of information is $\log c$.
} 


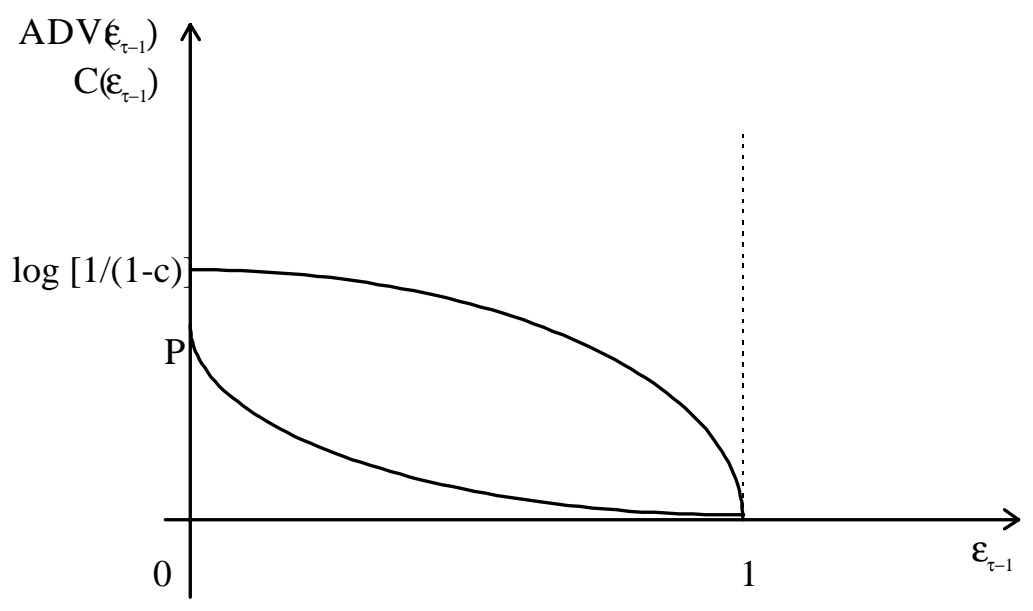

Figure 3: Dominance of uninformed traders.

uninformed traders dominate and informed traders vanish:

$$
\lim _{t \rightarrow \infty} \varepsilon_{t}^{I}=0 \quad \text { a.s. }
$$

Proof. See appendix.

Clearly, the lower is the power of the signal $P$, the lower is the threshold level for the flow cost $c$. In particular, when $P$ is close to zero ${ }^{22}$, any strictly positive information cost will cause uninformed traders to dominate and drive informed traders to extinction.

\subsection{Survival of Uninformed Traders}

This section contains the main result of our paper. We will show that, as long as the flow cost paid in order to obtain superior information is bounded away from zero, uninformed traders will not be driven to extinction by the informed. An intuitive reason for this result is that, as the wealth share of informed traders grows, the advantage given by superior information is gradually offset by its cost.

In particular, whether uninformed traders survive or not will crucially depend on the behaviour of the process of wealth accumulation in the neighbourhood of $\varepsilon_{t}^{I}=1$.

We can show that, when the evolutionary advantage of being informed is maximal - i.e. it coincides with its upper bound -, uninformed traders are not wiped out by the informed. A fortiori, uninformed traders survive when the evolutionary advantage of being informed is lower than its upper bound.

Let us consider the process of wealth accumulation when:

$$
A D V_{t}\left(\varepsilon_{t-1}^{I}\right)=\overline{A D V_{t}}\left(\varepsilon_{t-1}^{I}\right)
$$

\footnotetext{
${ }^{22}$ Recall that $P=0$ if: either signals and states of the world are independent, or the probability distribution over signals (states) is degenerate, so that only one signal (one state) occurs with positive probability.
} 


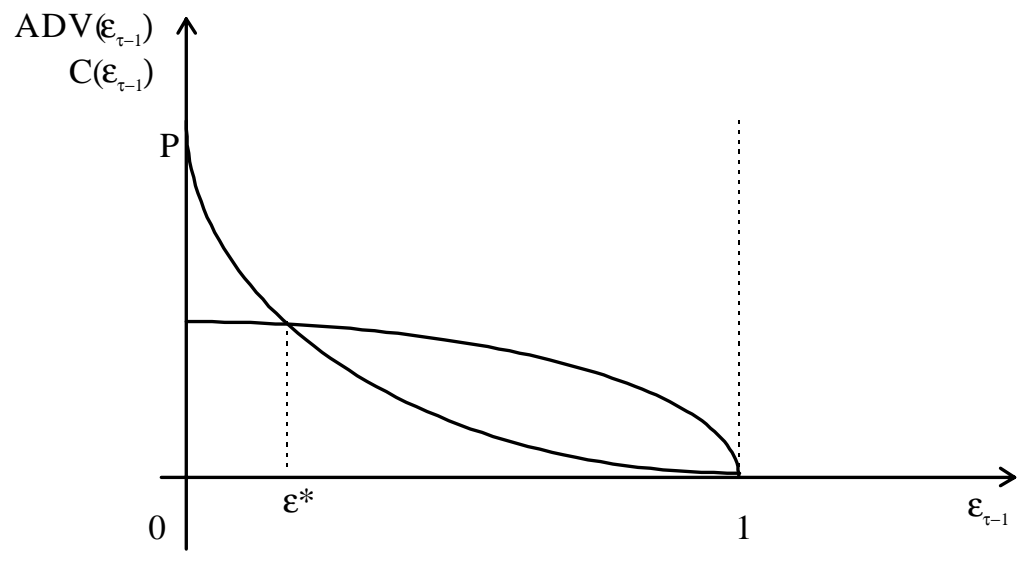

Figure 4: Survival of uninformed traders.

We have depicted this process in figure 4 . Call $\varepsilon^{*}$ the value for the wealth share of informed traders such that the advantage given by information is exactly offset by its cost. Formally $\varepsilon^{*}$ is defined as the solution (different from 1 ) of the following equation ${ }^{23}$ :

$$
A D V_{t}\left(\varepsilon_{t-1}^{I}\right)=C_{t}\left(\varepsilon_{t-1}^{I}\right)
$$

The properties we proved to hold for $\overline{A D V}_{t}$ and $C_{t}$ in the previous section guarantee that: as long as $c \leq\left(e^{P}-1\right) / e^{P}$ and $c$ is bounded away from zero, a solution for $\varepsilon^{*}$ always exists; it is unique ${ }^{24}$ and it is bounded away from 1.

We can study the behaviour of the process of wealth accumulation for informed traders observing that: for $\varepsilon_{t-1}^{I} \leq \varepsilon^{*}$, the process at $t$ behaves as a supermartingale; for $\varepsilon_{t-1}^{I} \geq \varepsilon^{*}$, the process at $t$ behaves as a submartingale. Formally:

$$
\begin{array}{ll}
E\left[\log \varepsilon_{t}^{I} \mid \Im_{t-1}\right] \geq \log \varepsilon_{t-1}^{I} & \text { if } \varepsilon_{t-1}^{I} \leq \varepsilon_{t}^{*} \\
E\left[\log \varepsilon_{t}^{I} \mid \Im_{t-1}\right] \leq \log \varepsilon_{t-1}^{I} & \text { if } \varepsilon_{t-1}^{I} \geq \varepsilon_{t}^{*}
\end{array}
$$

We can finally provide the main result of our analysis: we prove that uninformed traders survive with positive probability. The argument is by contradiction. Suppose that uninformed traders vanish almost surely: this would imply that their wealth share converges to zero with probability 1 . If this is the case, then there exists a time $T$ such that, after $T$, the process of wealth accumulation for informed traders behaves as a submartingale, i.e. it decreases on average. Therefore, the proof can be completed by showing that a bounded submartingale cannot converge almost surely to its upper bound ${ }^{25}$.

\footnotetext{
${ }^{23}$ The equation is clearly satisfied also by $\varepsilon_{t-1}^{I}=1$.

${ }^{24}$ Uniqueness is guaranteed by monotonicity of both functions.

${ }^{25}$ In order to prove that uninformed traders survive almost surely, we show in the appendix that the event $\varepsilon_{t}^{I} \rightarrow 1$ a.s. is contradicted on any set of sample paths with strictly positive measure.
} 
Proposition 17 (Survival of Uninformed Traders) Uninformed traders survive. Formally: $\lim \sup _{t \rightarrow \infty} \varepsilon_{t}^{U}>0$ almost surely.

Proof. See appendix.

Proposition 17 is the main result of the paper. When most traders invest in information, it pays for some of them to stay uninformed. So that even if the cost of information is small relatively to the quality of the information purchased, as measured by the power of the signal $P$, nevertheless traders who make their portfolio decisions on the basis of inferior information will survive. It is interesting to note that the result is not only due to the fact that, when more traders are informed, then prices reflect more of the purchased information ${ }^{26}$, but mainly to the fact that, in evolutionary terms, it does not pay to be informed when almost everyone else is. In other words, information is valuable as long as it stays "private" 27 .

We believe that this result can contribute to the strand of literature that attempts to explain the survival of noise traders.

Friedman [18]'s conjecture against the importance of noise traders in the process of price determination is therefore contradicted. It is too simplistic to argue that if noise traders are systematically wrong then they should make wrong decisions, incur losses and consequently disappear from the market. As long as the acquisition of superior information is a costly activity, uninformed traders will not be wiped out by the informed and will contribute to determine asset prices also in the long run.

\subsection{Low Cost Information}

In this section we examine the long run behaviour of the wealth shares of informed and uninformed traders as the flow cost that market participants pay in order to obtain superior information becomes more and more irrelevant.

When there is no cost in obtaining information, namely when $c=0$, then clearly traders who possess superior information dominate and all the others vanish. We ask under which conditions uninformed traders also vanish as $c \rightarrow 0$, so that we have continuity in the process of wealth accumulation.

We first perform some comparative statics exercises to ask what is the effect on the process of wealth accumulation of a reduction in the flow cost that market participants pay in order to obtain superior information. As usual we will distinguish between the two opposite components in the accumulation process and namely between what we called the cost and the evolutionary advantage of being informed.

When the flow cost $c$ decreases, the cost of being informed decreases as well. In fact:

$$
\frac{\partial C_{t}}{\partial c}=\frac{1-\varepsilon_{t-1}^{I}}{(1-c)\left(1-c \varepsilon_{t-1}^{I}\right)}>0
$$

In our diagram, the cost function shifts downwards.

\footnotetext{
${ }^{26}$ In fact, even traders who invest according to their priors would survive.

${ }^{27}$ In a different framework, Hirshleifer [23] develops a model where information is only imperfectly appropriable by its discoverer. He shows that risk-averse individuals might prefer not to obtain the information in the first place: a community of such individuals would actually pay something to an outsider not to reveal, in advance of market trading, which state will obtain.
} 


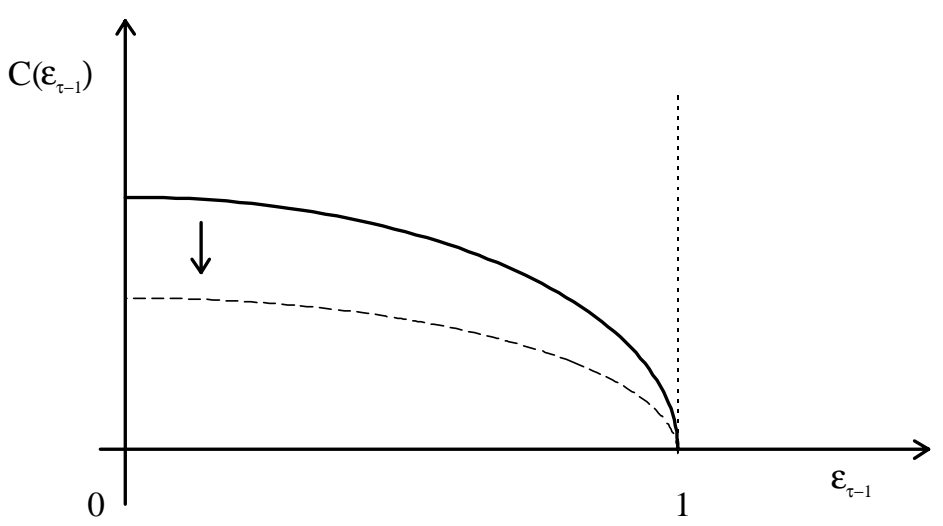

Figure 5: A decrease in the flow cost for information.

We need now to identify the effect of a reduction in the flow cost for information on the advantage of being informed. Recall that:

$$
A D V_{t}\left(\varepsilon_{t-1}^{I}\right)=\int_{0}^{\infty} \sum_{z=1}^{S} \theta_{z} \sum_{s=1}^{S} \frac{p_{s z}}{\theta_{z}} \log \frac{p_{s z} / \theta_{z}}{\gamma_{t-1} p_{s z} / \theta_{z}+\left(1-\gamma_{t-1}\right) p_{s t}^{U}} f\left(x_{t}\right) d x_{t}
$$

where

$$
\gamma_{t-1}\left(\varepsilon_{t-1}^{I}, c\right)=\frac{\varepsilon_{t-1}^{I}(1-c)}{\varepsilon_{t-1}^{I}(1-c)+\left(1-\varepsilon_{t-1}^{I}\right)}
$$

Therefore the advantage of being informed is given by the distance (as measured by the relative entropy) between conditional probabilities and $\gamma_{t-1} p_{s z} / \theta_{z}+\left(1-\gamma_{t-1}\right) p_{s t}^{U}$, which in fact represent respectively the portfolio of informed traders and the market portfolio. The factor $\gamma_{t-1}$ represents the wealth share of informed traders after information expenditure: it is the factor according to which informed traders determine market prices. The higher is $\gamma_{t-1}$, the higher is the weight of informed traders in the process of price determination, the smaller the distance between their portfolio and the market portfolio and, finally, the smaller the evolutionary advantage given by acquiring superior information.

$A$ decrease in the flow cost for information implies an increase in the factor $\gamma_{t-1}$ and therefore a decrease in $A D V_{t}$ :

$$
\frac{\partial \gamma_{t-1}}{\partial c}=\frac{-\varepsilon_{t-1}^{I}\left(1-\varepsilon_{t-1}^{I}\right)}{\left(1-c \varepsilon_{t-1}^{I}\right)^{2}}<0
$$

In our diagram, the advantage function shifts downwards.

We can summarise these comparative statics exercises claiming that a reduction in the flow cost for information has two opposite effects on the process of wealth accumulation of informed traders:

1. A direct positive effect through a reduction of $C_{t}\left(\varepsilon_{t-1}^{I}\right)$; 


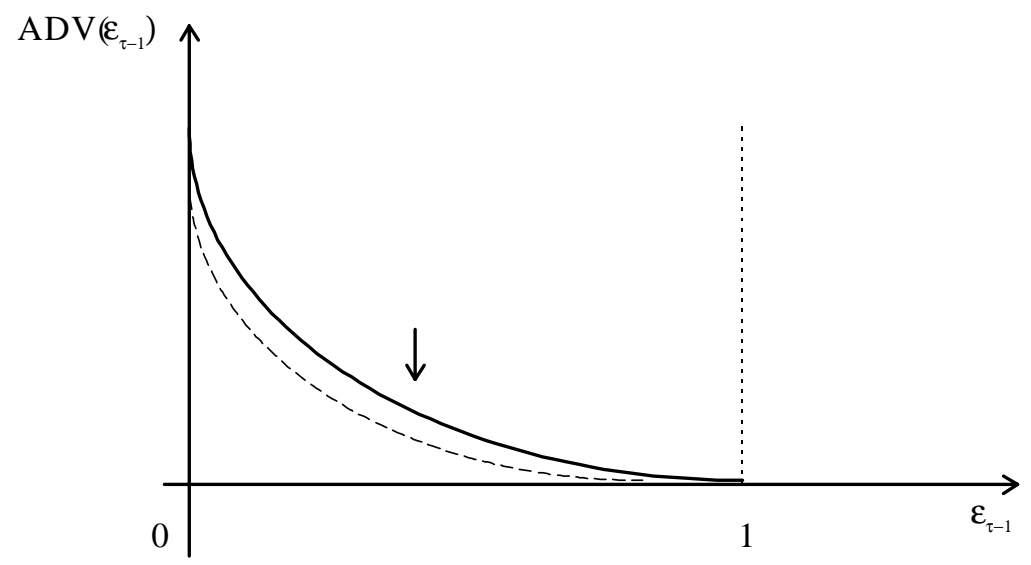

Figure 6: A decrease in the flow cost for information.

2. A negative indirect effect. A lower flow cost implies that informed traders have more influence on prices and market outcomes. Therefore the relative fitness of their behaviour with respect to the average market behaviour decreases.

As long as there is no full revelation, the direct positive effect prevails on the negative indirect effect, so that a decrease in the flow cost for information implies a larger value for $\varepsilon^{*}$. In the limit, as $c \rightarrow 0, \varepsilon^{*} \rightarrow 1$ and uninformed traders vanish.

Proposition 18 When $c \rightarrow 0$, as long as there is no full revelation, uninformed traders vanish:

$$
\lim _{c \rightarrow 0} \lim _{t \rightarrow \infty} \varepsilon_{t}^{I}=1
$$

Proof. See appendix.

\section{Full Revelation}

Most of the statements we proved in the previous sections assumed that the market never attains full revelation. It is therefore interesting to explore what is the long run outcome when we do, in fact, have full revelation. Corollary 9 shows that, as long as assumption 1 holds, prices are not fully revealing. Therefore, in order to have prices that fully reveal all the available information we need to relax our noise assumptions.

There are three different circumstances in which we might have full revelation in our setting: let us examine them one by one.

Case 1: $x_{t}$ known. 
Suppose that traders could actually observe the realisation of the random vector of asset supplies ${ }^{28}$, so that:

$$
\begin{aligned}
\Phi_{t}^{I} & =\left\{\rho_{t}, x_{t}, z\right\} \\
\Phi_{t}^{U} & =\left\{\rho_{t}, x_{t}\right\}
\end{aligned}
$$

If this is the case, then uninformed traders possess all the relevant information to infer from market prices the precise signal that informed traders observed. In fact the relation between market prices and signals observed, given the information set of uninformed traders, will be one to one.

\section{Case 2: $P=E_{\theta}$.}

The power of the signal $P$ is maximal when it is equal to the unexpectedness (the entropy) of the signal, $E_{\theta}$. In this case, signals and states are perfectly correlated and informed traders know the actual state of the world when they observe the signal. They therefore do not demand any other asset than the one which is going to pay with probability one. As a result, uninformed traders are able to extract fully this information from market prices.

Case 3: $P=0$.

The power of the signal $P$ is null when signals and states are independent or when the marginal probability distribution over the signal space (or over the state space) is degenerate so that one signal (or one state) occurs with probability one. In this case, signals do not convey relevant information on the states of the world and therefore there is nothing to infer from market prices. However, if we think of full revelation as of the situation in which the posterior of informed and uninformed traders coincide, then this case falls in that category since the posteriors of both types of traders are equal to their common prior.

Whenever full revelation occurs the dynamics of the wealth shares become trivial. In fact traders invest according to the same portfolio rule and therefore the only difference in their wealth accumulation rates is given by the flow cost $c$ paid for information acquisition by informed traders. As long as $c>0$, the wealth growth rate of informed traders will be outperformed by the wealth growth rate of the uninformed. Uninformed traders will dominate and informed traders will vanish. In the long run no information will be purchased and traders will invest according to their priors.

It is interesting to notice that this is an efficient outcome in case 3 , where the costly information available is not valuable. However, this is not the efficient outcome in cases 1 and 2, where some relevant information is available and yet not exploited. In particular the case $P=E_{\theta}$ is surprising: when the costly information available is perfect, then the private return of being informed becomes null and the information itself is not exploited ${ }^{29}$. Efficient markets theorists have claimed that "at any time prices fully reflect all available

\footnotetext{
${ }^{28}$ For full revelation to occur it suffices that $x_{t}$ is included in the information set of uninformed traders. However, to minimise the informational asymmetry across traders, we keep the assumption that the information sets of the two types of traders only differ because of the signal $z$.

${ }^{29}$ Berk [2] constructs an example where, even if the equilibrium is fully revealing, then agents might still want to pay a price for information. This is obtained, however, in a multiperiod model where information is fully revealed only in the last period and traders not only choose whether to acquire information or not, but also the timing of the purchase of information.
} 


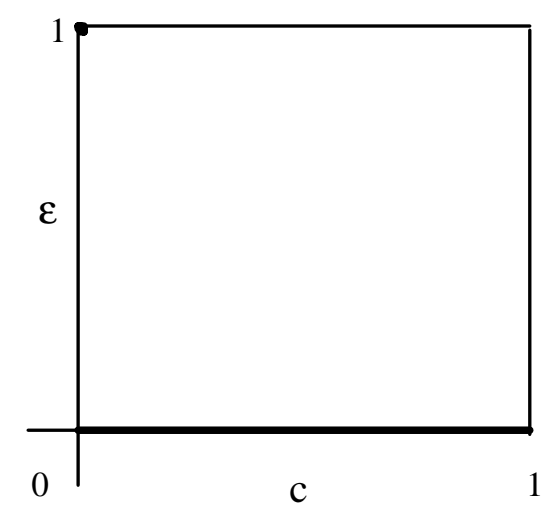

Figure 7: Full revelation.

information" (Fama [15], p. 383). In our setting the efficient markets hypothesis is clearly violated and it emerges, like in Grossman and Stiglitz [21], that costless information is not only a sufficient condition for market efficiency, but also a necessary one.

In fact, if we assume that information is available at a zero cost, then clearly everyone informed is the only stable equilibrium and, as a result, markets are informationally efficient. However, costless information is our only chance to achieve informational efficiency, as markets fail to be efficient as soon as we depart from the assumption of costless information.

As Grossman and Stiglitz put it: "Efficient markets theorists seem to be aware that costless information is a sufficient condition for prices to fully reflect all available information [...]; they are not aware that it is a necessary condition" ([21], p. 404).

Moreover, since in the presence of full revelation informed traders vanish for any strictly positive $c$, the efficient market hypothesis fails also if $c \rightarrow 0$. As a result, the claim that when information is costless markets are efficient is not robust to small changes in the information costs. As the diagram in figure 7 shows, there is a discontinuity in the process of wealth accumulation when there is full revelation and $c=0$. In fact:

$$
\lim _{c \rightarrow 0} \lim _{t \rightarrow \infty} \varepsilon_{t}^{I}(c)=0
$$

while

$$
\left.\lim _{t \rightarrow \infty} \varepsilon_{t}^{I}(c)\right|_{c=0}=1
$$




\section{Concluding Remarks}

In the evolutionary setting for a financial market developed by Blume and Easley [6], we consider an infinitely repeated version of a model à la Grossman and Stiglitz [21] with asymmetrically informed traders.

Informed traders observe the realisation of a payoff relevant signal before making their portfolio decisions. Uninformed traders do not have direct access to this kind of information, but can partially infer it from market prices. As a counterpart for their privileged information, informed traders pay a flow cost. As a result, information acquisition triggers a trade-off in our setting. We prove that, as long as information is costly, a strictly positive measure of uninformed traders will survive.

This result contributes to the literature on noise trading. It suggests that Friedman [18]'s argument against the importance of noise traders in the process of price determination is too simplistic. Traders whose beliefs are "wrong" according to the best available information, in fact, are not wiped out by market forces and do affect asset prices in the long run.

\section{APPENDIX}

Proof of Proposition 5 (Existence) Equilibrium prices must satisfy:

$$
\rho_{s t}=\left[(1-c) \varepsilon_{t-1} \frac{p_{s z}}{\theta_{z}}+\left(1-\varepsilon_{t-1}\right) \sum_{z^{\prime}} \frac{p_{s z^{\prime}}}{\theta_{z^{\prime}}} \cdot \frac{\theta_{z^{\prime}} f\left(x_{t}^{z^{\prime}}\right)}{\sum_{z^{\prime \prime}} \theta_{z^{\prime \prime}} f\left(x_{t}^{z^{\prime \prime}}\right)}\right] \frac{w_{t-1}}{x_{s t}}
$$

where

$$
x_{t}^{z}=\left[(1-c) \varepsilon_{t-1}^{I} \frac{p_{s z}}{\theta_{z}}+\left(1-\varepsilon_{t-1}^{I}\right) p_{s t}^{U}\left(\rho_{t}\right)\right] \frac{w_{t-1}}{\rho_{s t}}
$$

Assuming that $p_{s t}^{U}\left(\rho_{t}\right)$ is continuous in equilibrium prices and excluding the zero probability event $\rho_{s t}=0$, we find that $x_{s t}^{z}$ is continuous in $\rho_{s t}$. By continuity of the density function and boundedness of beliefs, we can conclude that an equilibrium exists and the posterior of uninformed traders is indeed continuous in equilibrium prices. As a result, a vector of equilibrium prices exists.

Proof of Proposition 6 (Uniqueness) By definition, the mapping $\varphi_{t}\left(p_{t}^{U}\right)$ is a contraction if $\exists \nu<1$ such that, $\forall p_{t}^{U}, g_{t}^{U} \in \mathcal{P}_{t}$ :

$$
\left\|\varphi_{t}\left(p_{t}^{U}\right)-\varphi_{t}\left(g_{t}^{U}\right)\right\| \leq \nu\left\|p_{t}^{U}-g_{t}^{U}\right\|
$$

Consider now that:

$$
\begin{aligned}
\varphi_{s t}\left(p_{t}^{U}\right)-\varphi_{s t}\left(g_{t}^{U}\right) & =\sum_{z} p_{s z}\left[\frac{1}{\sum_{z^{\prime}} \theta_{z^{\prime}} r_{t}\left(z, z^{\prime}, p_{t}^{U}\right)}-\frac{1}{\sum_{z^{\prime}} \theta_{z^{\prime}} r_{t}\left(z, z^{\prime}, g_{t}^{U}\right)}\right]= \\
& =\sum_{z} p_{s z} \frac{\sum_{z^{\prime}} \theta_{z^{\prime}}\left[r_{t}\left(z, z^{\prime}, g_{t}^{U}\right)-r_{t}\left(z, z^{\prime}, p_{t}^{U}\right)\right]}{\sum_{z^{\prime}} \theta_{z^{\prime}} r_{t}\left(z, z^{\prime}, p_{t}^{U}\right) \sum_{z^{\prime}} \theta_{z^{\prime}} r_{t}\left(z, z^{\prime}, g_{t}^{U}\right)}
\end{aligned}
$$

Notice that, since $\forall z, p_{t}^{U}$ :

$$
\frac{\theta_{z}}{\sum_{z^{\prime}} \theta_{z^{\prime}} r_{t}\left(z, z^{\prime}, p_{t}^{U}\right)} \leq 1
$$


then clearly, $\forall z, p_{t}^{U}$ :

$$
\sum_{z^{\prime}} \theta_{z^{\prime}} r_{t}\left(z, z^{\prime}, p_{t}^{U}\right) \geq \theta_{z}
$$

As a result:

$$
\left|\varphi_{s t}\left(p_{t}^{U}\right)-\varphi_{s t}\left(g_{t}^{U}\right)\right| \leq\left|\sum_{z} \frac{p_{s z}}{\theta_{z}} \cdot \frac{\sum_{z^{\prime}} \theta_{z^{\prime}}\left[r_{t}\left(z, z^{\prime}, g_{t}^{U}\right)-r_{t}\left(z, z^{\prime}, p_{t}^{U}\right)\right]}{\theta_{z}}\right|
$$

Finally, by subadittivity of the supremum norm:

$$
\left\|\varphi_{t}\left(p_{t}^{U}\right)-\varphi_{t}\left(g_{t}^{U}\right)\right\| \leq \sum_{z} \frac{p_{s z}}{\theta_{z}} \cdot \frac{\sum_{z^{\prime}} \theta_{z^{\prime}}\left\|r_{t}\left(z, z^{\prime}, g_{t}^{U}\right)-r_{t}\left(z, z^{\prime}, p_{t}^{U}\right)\right\|}{\theta_{z}}
$$

The condition:

$$
\left\|r_{t}\left(z, z^{\prime}, p_{t}^{U}\right)-r_{t}\left(z, z^{\prime}, g_{t}^{U}\right)\right\| \leq \nu \theta_{z}\left\|p_{t}^{U}-g_{t}^{U}\right\|
$$

implies:

$$
\left\|\varphi_{t}\left(p_{t}^{U}\right)-\varphi_{t}\left(g_{t}^{U}\right)\right\| \leq \sum_{z} \frac{p_{s z}}{\theta_{z}} \cdot \frac{\sum_{z^{\prime}} \theta_{z^{\prime}} \nu \theta_{z}\left\|p_{t}^{U}-g_{t}^{U}\right\|}{\theta_{z}}=\nu\left\|p_{t}^{U}-g_{t}^{U}\right\|
$$

and therefore $\varphi_{t}\left(p_{t}^{U}\right)$ is a contraction mapping by definition.

Proof of Corollary 9 (Non-Fully Revealing Equilibrium) Recall that we denoted by:

$$
q_{z t}=\frac{\theta_{z} f\left(x_{t}^{z}\right)}{\sum_{z^{\prime}} \theta_{z^{\prime}} f\left(x_{t}^{z^{\prime}}\right)}
$$

the probability that uninformed traders attach to signal $z$, after observing market prices. Call $\hat{z}$ the "true" value of $z$. For full revelation to occur at date $t$, we need that:

$$
q_{z t}=\left\{\begin{array}{l}
1 \text { for } z=\hat{z} \\
0 \text { for } z \neq \hat{z}
\end{array}\right\}
$$

For likelihood ratios this requires, $\forall z^{\prime}$ :

$$
\frac{f\left(x_{t}^{z^{\prime}}\right)}{f\left(x_{t}^{z}\right)}=\left\{\begin{array}{c}
0 \text { for } z=\widehat{z} \\
\infty \text { for } z \neq \hat{z}
\end{array}\right\}
$$

which cannot be obtained with positive probability as long as $f\left(x_{t}\right)$ has full support.

Proof of Lemma 11 Lemmas A.1. and A.2 in [6] guarantee that on the sample paths where

$$
\lim _{t \rightarrow \infty} \inf \sum_{\tau=1}^{t} E\left[\lambda_{\tau}^{i} \mid \Im_{\tau-1}\right]>-\infty
$$

then, almost surely:

$$
\sum_{\tau=1}^{t} \operatorname{Var}\left[\lambda_{\tau}^{i} \mid \Im_{\tau-1}\right]<\infty
$$


and corollary 4.5 in [16] proves that, on 15:

$$
\lim _{t \rightarrow \infty} \inf \sum_{\tau=1}^{t} \lambda_{\tau}^{i}<-\infty \text { a.s. }
$$

As a result, 14 implies 16 and therefore trader $i$ dominates by definition.

Proof of Proposition 13 (Upper bound) We prove this proposition in two steps. We first show that:

$$
\sum_{z=1}^{S} \theta_{z} \sum_{s} \frac{p_{s z}}{\theta_{z}} \log \frac{p_{s z} / \theta_{z}}{p_{s}} \geq E\left[\sum_{z=1}^{S} \theta_{z} \sum_{s} \frac{p_{s z}}{\theta_{z}} \log \frac{p_{s z} / \theta_{z}}{p_{s t}^{U}\left(\rho_{t}\right)}\right]
$$

and then we prove that 17 implies our claim. Equation 17 can be interpreted as claiming that, on average, posteriors are closer to the "true" distribution over the state space, than priors. This is guaranteed by maximum likelihood. In fact, if $z$ is the "true" signal that has been observed, then it follows ${ }^{30}$ that

$$
z=\arg \max _{z^{\prime}} E\left[\log f\left(x_{t}^{z^{\prime}}\right)\right]
$$

so that, $\forall z^{\prime}\{1, \ldots, S\}$ :

$$
E\left[\log f\left(x_{t}^{z}\right)\right] \geq E\left[\log f\left(x_{t}^{z^{\prime}}\right)\right]
$$

and therefore:

$$
E\left[\log \frac{f\left(x_{t}^{z}\right)}{f\left(x_{t}^{z^{\prime}}\right)}\right] \geq 0
$$

By concavity:

$$
\log E\left[\frac{f\left(x_{t}^{z}\right)}{f\left(x_{t}^{z^{\prime}}\right)}\right] \geq E\left[\log \frac{f\left(x_{t}^{z}\right)}{f\left(x_{t}^{z^{\prime}}\right)}\right] \geq 0
$$

and finally:

$$
E\left[\frac{f\left(x_{t}^{z}\right)}{f\left(x_{t}^{z^{\prime}}\right)}\right] \geq 1
$$

As a result:

$$
E\left[\frac{\theta_{z} f\left(x_{t}^{z}\right)}{\sum_{z^{\prime}} \theta_{z^{\prime}} f\left(x_{t}^{z^{\prime}}\right)}\right] \geq \theta_{z}
$$

Now, notice that the left hand side of equation 18 is the weight that, on average is attached to the "real" conditional probability $p_{s z} / \theta_{z}$ by traders' posterior, while $\theta_{z}$ is the weight that is attached to the same "true" probability by traders' prior. In fact:

$$
\begin{aligned}
p_{s t}^{U} & =\sum_{z} \frac{p_{s z}}{\theta_{z}} \cdot \frac{\theta_{z} f\left(x_{t}^{z}\right)}{\sum_{z^{\prime}} \theta_{z^{\prime}} f\left(x_{t}^{z^{\prime}}\right)} \\
p_{s} & =\sum_{z} \frac{p_{s z}}{\theta_{z}} \cdot \theta_{z}
\end{aligned}
$$

\footnotetext{
${ }^{30}$ See, for example, Cramer [10].
} 
Therefore, on average, the posterior attaches a higher weight to the observed signal than the prior. As a consequence, the average distance, as measured by relative entropy, between the "true" distribution and the posterior is smaller than the average distance between the "true" distribution and the prior ${ }^{31}$. This proves 17 and constitutes the first step of our proof. Next we show that 17 implies our claim. In fact equation 17 can be rewritten as follows:

$$
\sum_{z=1}^{S} \theta_{z} \sum_{s} \frac{p_{s z}}{\theta_{z}} \log \frac{p_{s}}{p_{s z} / \theta_{z}} \leq E\left[\sum_{z=1}^{S} \theta_{z} \sum_{s} \frac{p_{s z}}{\theta_{z}} \log \frac{p_{s t}^{U}}{p_{s z} / \theta_{z}}\right]
$$

and by simple algebra:

$$
\begin{aligned}
& \sum_{z=1}^{S} \theta_{z} \sum_{s} \frac{p_{s z}}{\theta_{z}} \log \left[\frac{\left(1-\gamma_{t-1}\right) p_{s}}{p_{s z} / \theta_{z}}+\frac{\gamma_{t-1} p_{s z} / \theta_{z}}{p_{s z} / \theta_{z}}\right] \leq \\
\leq & E\left[\sum_{z=1}^{S} \theta_{z} \sum_{s} \frac{p_{s z}}{\theta_{z}} \log \left(\frac{\left(1-\gamma_{t-1}\right) p_{s t}^{U}}{p_{s z} / \theta_{z}}+\frac{\gamma_{t-1} p_{s z} / \theta_{z}}{p_{s z} / \theta_{z}}\right)\right] \\
& \sum_{z=1}^{S} \theta_{z} \sum_{s} \frac{p_{s z}}{\theta_{z}} \log \frac{\gamma_{t-1} p_{s z} / \theta_{z}+\left(1-\gamma_{t-1}\right) p_{s}}{p_{s z} / \theta_{z}} \leq \\
\leq & E\left[\sum_{z=1}^{S} \theta_{z} \sum_{s} \frac{p_{s z}}{\theta_{z}} \log \frac{\gamma_{t-1} p_{s z} / \theta_{z}+\left(1-\gamma_{t-1}\right) p_{s t}^{U}}{p_{s z} / \theta_{z}}\right]
\end{aligned}
$$

which finally proves that:

$$
A D V_{t}\left(\varepsilon_{t-1}^{I}\right) \leq \sum_{z=1}^{S} \theta_{z} \sum_{s} \frac{p_{s z}}{\theta_{z}} \log \frac{p_{s z} / \theta_{z}}{\gamma_{t-1} p_{s z} / \theta_{z}+\left(1-\gamma_{t-1}\right) p_{s}}
$$

Proof of Proposition 14 We can show that:

$$
\frac{\partial \overline{A D V}_{t}\left(\varepsilon_{t-1}^{I}\right)}{\partial \varepsilon_{t-1}^{I}} \leq 0
$$

Recall that:

$$
\gamma_{t-1}=\frac{\varepsilon_{t-1}^{I}(1-c)}{1-c \varepsilon_{t-1}^{I}}
$$

and therefore:

$$
\frac{\partial \gamma_{t-1}}{\partial \varepsilon_{t-1}^{I}}=\frac{1-c}{\left(1-c \varepsilon_{t-1}^{I}\right)^{2}} \geq 0
$$

\footnotetext{
${ }^{31}$ This can be formally shown checking that the relative entropy is indeed decreasing in the weight attached to the "true" probability distribution.
} 
The first derivative of $\overline{A D V}_{t}\left(\varepsilon_{t-1}^{I}\right)$ with respect to $\varepsilon_{t-1}^{I}$ can be computed as:

$$
\frac{\partial \overline{A D V}_{t}\left(\varepsilon_{t-1}^{I}\right)}{\partial \varepsilon_{t-1}^{I}}=-\frac{\partial \gamma_{t-1}}{\partial \varepsilon_{t-1}^{I}} \cdot \sum_{z} \theta_{z} \sum_{s} \frac{p_{s z}}{\theta_{z}} \cdot \frac{p_{s z} / \theta_{z}-p_{s}}{\gamma_{t-1} p_{s z} / \theta_{z}-\left(1-\gamma_{t-1}\right) p_{s}}
$$

We can show that the expression:

$$
\sum_{z} \theta_{z} \sum_{s} \frac{p_{s z}}{\theta_{z}} \cdot \frac{p_{s z} / \theta_{z}-p_{s}}{\gamma_{t-1} p_{s z} / \theta_{z}-\left(1-\gamma_{t-1}\right) p_{s}}
$$

is always non-negative, so that our claim on the first derivative follows. Let

$$
\begin{aligned}
& A=\sum_{z} \theta_{z} \sum_{s} \frac{p_{s z}}{\theta_{z}} \cdot \frac{p_{s z} / \theta_{z}}{\gamma_{t-1} p_{s z} / \theta_{z}-\left(1-\gamma_{t-1}\right) p_{s}} \\
& B=\sum_{z} \theta_{z} \sum_{s} \frac{p_{s z}}{\theta_{z}} \cdot \frac{p_{s}}{\gamma_{t-1} p_{s z} / \theta_{z}-\left(1-\gamma_{t-1}\right) p_{s}}
\end{aligned}
$$

so that equation 19 can be rewritten as follows:

$$
A-B
$$

Take, now, $\log A$. By concavity:

$$
\log A \geq \sum_{z} \theta_{z} \sum_{s} \frac{p_{s z}}{\theta_{z}} \log \frac{p_{s z} / \theta_{z}}{\gamma_{t-1} p_{s z} / \theta_{z}-\left(1-\gamma_{t-1}\right) p_{s}} \geq 0
$$

This implies that $A \geq 1$. Notice now that:

$$
\gamma_{t-1} A+\left(1-\gamma_{t-1}\right) B=1
$$

where $0 \leq \gamma_{t-1} \leq 1$. As a result, $A \geq 1 \Rightarrow B \leq 1$ and finally $A-B \geq 0$. This, together with $\partial \gamma_{t-1} / \partial \varepsilon_{t-1}^{I} \geq 0$ proves that $\overline{A D V}_{t}\left(\varepsilon_{t-1}^{I}\right)$ is a monotonically decreasing function.

Proof of Proposition 15 We can show that the upper bound of $A D V_{t}\left(\varepsilon_{t-1}^{I}\right)$ is flat at $\varepsilon_{t-1}^{I}=1$. A fortiori, $A D V_{t}\left(\varepsilon_{t-1}^{I}\right)$ is flat. Recall that:

$$
\frac{\partial \overline{A D V}_{t}\left(\varepsilon_{t-1}^{I}\right)}{\partial \varepsilon_{t-1}^{I}}=-\frac{\partial \gamma_{t-1}}{\partial \varepsilon_{t-1}^{I}} \cdot \sum_{z} \theta_{z} \sum_{s} \frac{p_{s z}}{\theta_{z}} \cdot \frac{p_{s z} / \theta_{z}-p_{s}}{\gamma_{t-1} p_{s z} / \theta_{z}-\left(1-\gamma_{t-1}\right) p_{s}}
$$

where:

$$
\gamma_{t-1}=\frac{\varepsilon_{t-1}^{I}(1-c)}{1-c \varepsilon_{t-1}^{I}} \quad \text { and } \quad \frac{\partial \gamma_{t-1}}{\partial \varepsilon_{t-1}^{I}}=\frac{1-c}{\left(1-c \varepsilon_{t-1}^{I}\right)^{2}}
$$

so that:

$$
\lim _{\varepsilon_{t-1}^{I} \rightarrow 1} \gamma_{t-1}=1 \quad \text { and } \quad \lim _{\varepsilon_{t-1}^{I} \rightarrow 1} \frac{\partial \gamma_{t-1}}{\partial \varepsilon_{t-1}^{I}}=\frac{1}{1-c}
$$


Finally:

$$
\lim _{\varepsilon_{t-1}^{I} \rightarrow 1} \frac{\partial \overline{A D V}_{t}\left(\varepsilon_{t-1}^{I}\right)}{\partial \varepsilon_{t-1}^{I}}=-\frac{1}{1-c} \cdot \sum_{z} \theta_{z} \sum_{s} \frac{p_{s z}}{\theta_{z}} \cdot \frac{p_{s z} / \theta_{z}-p_{s}}{p_{s z} / \theta_{z}}=0
$$

Proof of Proposition 16 (Dominance of Uninformed Traders) Dominance of uninformed traders is an immediate consequence of lemma 11. The fact that the wealth share of informed traders is a supermartingale implies that:

$$
E\left[\lambda_{t}^{I} \mid \Im_{t-1}\right]=A D V_{t}\left(\varepsilon_{t-1}^{I}\right)-C_{t}\left(\varepsilon_{t-1}\right) \leq 0
$$

Notice that:

$$
\varepsilon_{t-1}^{I} \lambda_{t}^{I}+\left(1-\varepsilon_{t-1}^{I}\right) \lambda_{t}^{U}=1
$$

so that 20 implies:

$$
E\left[\lambda_{t}^{U} \mid \Im_{t-1}\right] \geq 0
$$

As a result:

$$
\sum_{t=1}^{\infty} E\left[\lambda_{t}^{U} \mid \Im_{t-1}\right] \geq 0
$$

and by lemma 11 uninformed traders dominate: $\lim _{t \rightarrow \infty} \varepsilon_{t}^{U}>0$ almost surely. We can now show that uninformed traders will determine asset prices asymptotically. By the convergence theorem for supermartingales we know that:

$$
\operatorname{Pr}\left\{\omega \in \Omega\left|\exists \gamma^{*}(\omega)\right| \forall \xi>0, \exists T, \forall t \geq T,\left|\log \varepsilon_{t}^{I}(\omega)-\gamma^{*}(\omega)\right|<\xi\right\}=1
$$

or equivalently, $\log \varepsilon_{t}^{I} \rightarrow \gamma^{*}$ almost surely. Call $A \subseteq \Omega$ the set of sample paths such that $\log \varepsilon_{t}^{I}$ converges; clearly also $\varepsilon_{t}^{U}$ converges on $A$. As a result, $\forall \omega \in A$ :

$$
\frac{\varepsilon_{t}^{U}(\omega)}{\varepsilon_{t-1}^{U}(\omega)}=\frac{p_{s t}^{U}}{x_{s t} \rho_{s t} / w_{t-1}} \rightarrow 1
$$

Since, as we just showed, $\varepsilon_{t}^{U}(\omega) \nrightarrow 0$, this implies that:

$$
x_{s t} \rho_{s t} / w_{t-1} \rightarrow p_{s t}^{U} \text { a.s. }
$$

We can now show that informed traders are driven to extinction by the uninformed. Let us go back to the market clearing condition:

$$
\frac{x_{s t} \rho_{s t}}{w_{t-1}}=(1-c) \varepsilon_{t-1}^{I} \frac{p_{s z}}{\theta_{z}}+\left(1-\varepsilon_{t-1}^{I}\right) p_{s t}^{U}
$$

Because of no full revelation $p_{s t}^{U} \nrightarrow(1-c) p_{s z} / \theta_{z}$ and therefore necessarily $\varepsilon_{t}^{I} \rightarrow 0$ a.s.

Lemma $19 A$ bounded supermartingale $\varepsilon_{t}$, such that, $\forall t \geq 0$ :

- $0 \leq \varepsilon_{t} \leq 1$ 
- $E\left[\varepsilon_{t} \mid \varepsilon_{t-1}\right] \leq \varepsilon_{t-1}$

- $\varepsilon_{0}<1$

cannot converge a.s. to its upper bound.

Proof of Lemma 19 Suppose, by contradiction, that

$$
\lim _{t \rightarrow \infty} \varepsilon_{t}=1 \text { a.s. }
$$

Almost sure convergence implies convergence in probability. Since $\varepsilon_{t}$ is a bounded random variable, convergence in probability implies convergence in mean. Therefore:

$$
E\left[\varepsilon_{t}\right] \rightarrow 1
$$

However, we can show that $E\left[\varepsilon_{t}\right] \leq \varepsilon_{0}$. In fact, by iterated expectation:

$$
\begin{aligned}
E\left[\varepsilon_{t} \mid \mathfrak{F}_{\mathbf{0}}\right] & =E\left[\varepsilon_{t}\left|\mathfrak{F}_{t-1}\right| \mathfrak{F}_{0}\right] \leq E\left[\varepsilon_{t-1} \mid \mathfrak{F}_{0}\right] \leq \\
& \leq \ldots \leq E\left[\varepsilon_{\mathbf{0}} \mid \mathfrak{F}_{\mathbf{0}}\right]=\varepsilon_{\mathbf{0}}
\end{aligned}
$$

As a result:

$$
E\left[\varepsilon_{t} \mid \mathfrak{F}_{0}\right] \leq \varepsilon_{\mathbf{0}}
$$

and

$$
E\left[E\left[\varepsilon_{t} \mid \mathfrak{F}_{0}\right]\right]=E\left[\varepsilon_{t}\right] \leq \varepsilon_{0}
$$

which contradicts

$$
E\left[\varepsilon_{t}\right] \rightarrow 1
$$

Proof of Proposition 17 (Survival of Uninformed Traders) Suppose, by contradiction, that uninformed traders are driven to extinction with probability 1, i.e. :

$$
\lim _{t \rightarrow \infty} \varepsilon_{t}^{I}=1 \text { a.s. }
$$

If this is the case, then clearly, with probability $1, \lim _{t \rightarrow \infty} \varepsilon_{t}^{I}>\varepsilon^{*}$. However, for $\varepsilon_{t}^{I}>\varepsilon^{*}$ the process of wealth accumulation for informed traders behaves as a supermartingale and, because of lemma 19, it cannot almost surely converge to its upper bound. This contradiction shows that uninformed traders survive with strictly positive probability:

$$
\operatorname{Pr}\left\{\lim \sup _{t \rightarrow \infty} \varepsilon_{t}^{U}>0\right\}>0
$$

We can now show that $\nexists Q \subseteq \Im$ s.t. $\operatorname{Pr}\{Q\}>0$ and $\lim _{t \rightarrow \infty} \varepsilon_{t}^{I}=1$ a.s. on $Q$, so that clearly survival of uninformed traders occurs with probability one. Take, in fact, any $Q \subseteq \Im$ s.t. $\operatorname{Pr}\{Q\}>0$ and assume, by contradiction, that $\lim _{t \rightarrow \infty} \varepsilon_{t}^{I}=1$ a.s. on $Q$. Consider the natural filtration on $Q$ :

$$
Q_{0} \subseteq Q_{1} \subseteq \ldots \subseteq Q_{t} \subseteq \ldots \subseteq Q
$$


Call $g\left(x_{t}\right)$ the joint density of $\left\{x_{s t}\right\}_{s=1}^{S}$ on $Q$ and $\left\{\left\{p_{s z}^{\prime}\right\}_{s=1}^{S}\right\}_{z=1}^{S}$ the joint probability of $(s, z)$ on $Q$. Recall that $(s, z)$ is i.i.d., so that, on any $Q$ with strictly positive measure, $\exists T \mid \forall t \geq T, \operatorname{Pr}\left\{(s, z) \mid Q_{t-1}\right\}=p_{s z}$. As a result, $\forall t \geq T$ :

$$
\begin{aligned}
E\left[\log \varepsilon_{t}^{I} \mid Q_{t-1}\right]= & \int_{0}^{\infty} \sum_{z=1}^{S} \theta_{z} \sum_{s=1}^{S} \frac{p_{s z}}{\theta_{z}} \log \frac{p_{s z} / \theta_{z}}{\gamma_{t-1}\left(p_{s z} / \theta_{z}\right)+\left(1-\gamma_{t-1}\right) p_{s t}^{U}} g\left(x_{t}\right) d x_{t}+ \\
& +\log \frac{1-c}{1-c \varepsilon_{t-1}^{I}}+\log \varepsilon_{t-1}^{I}
\end{aligned}
$$

Let:

$$
A D V_{t}\left(\varepsilon_{t-1}^{I} \mid Q\right) \equiv \int_{0}^{\infty} \sum_{z=1}^{S} \theta_{z} \sum_{s=1}^{S} \frac{p_{s z}}{\theta_{z}} \log \frac{p_{s z} / \theta_{z}}{\gamma_{t-1}\left(p_{s z} / \theta_{z}\right)+\left(1-\gamma_{t-1}\right) p_{s t}^{U}} g\left(x_{t}\right) d x_{t}
$$

As above, we can show that, $\forall g\left(x_{t}\right)$ :

$$
A D V_{t}\left(\varepsilon_{t-1}^{I} \mid Q\right) \geq 0
$$

In particular: $A D V_{t}\left(\varepsilon_{t-1}^{I}=0 \mid Q\right)=P$ and $A D V_{t}\left(\varepsilon_{t-1}^{I}=1 \mid Q\right)=0$. Moreover:

$$
\lim _{\varepsilon_{t}^{I} \rightarrow 1} \frac{\partial A D V_{t}\left(\varepsilon_{t-1}^{I} \mid Q\right)}{\partial \varepsilon_{t-1}^{I}}=0
$$

so that $A D V_{t}\left(\varepsilon_{t-1}^{I} \mid Q\right)$ is flat at $\varepsilon_{t-1}^{I}=1$. By similar arguments as above, we can then show that $\varepsilon_{t}^{I} \rightarrow 1$ a.s. on $Q$ entails a contradiction. As a result, $\forall Q \subseteq \Im$ s. t. $\operatorname{Pr}\{Q\}>0$ :

$$
\limsup _{t \rightarrow \infty} \varepsilon_{t}^{U}>0 \quad \text { a.s. on } Q
$$

which clearly implies:

$$
\limsup _{t \rightarrow \infty} \varepsilon_{t}^{U}>0 \quad \text { a.s. on } \Im
$$

Proof of Proposition 18 Unless we attain full revelation:

$$
\lim _{c \rightarrow \mathbf{0}} A D V_{t}\left(\varepsilon_{t-1}^{I}\right)>0
$$

and

$$
\lim _{c \rightarrow 0} C_{t}\left(\varepsilon_{t-1}^{I}\right)=0
$$

so that, as $c \rightarrow 0$ :

$$
E\left[\log \varepsilon_{t}^{I} \mid \Im_{t-1}\right] \geq \log \varepsilon_{t-1}^{I}
$$

and $\log \varepsilon_{t}^{I}$ is a submartingale. Dominance of informed traders is a consequence of lemma 11 . The fact that the wealth share of informed traders is a submartingale implies that:

$$
E\left[\lambda_{t}^{I} \mid \Im_{t-1}\right]=A D V_{t}\left(\varepsilon_{t-1}^{I}\right)-C_{t}\left(\varepsilon_{t-1}\right) \geq 0
$$


As a result:

$$
\sum_{t=1}^{\infty} E\left[\lambda_{t}^{I} \mid \Im_{t-1}\right] \geq 0
$$

and by lemma 11 informed traders dominate: $\lim _{t \rightarrow \infty} \varepsilon_{t}^{I}>0$ almost surely. We can now show that informed traders will determine asset prices asymptotically. By the convergence theorem for submartingales we know that almost surely $\varepsilon_{t}^{I}$ converges. As a result, on a set with measure one:

$$
\frac{\varepsilon_{t}^{I}(\omega)}{\varepsilon_{t-1}^{I}(\omega)}=\frac{(1-c) p_{s z} / \theta_{z}}{x_{s t} \rho_{s t} / w_{t-1}} \rightarrow 1
$$

Since, as we just argued, $\varepsilon_{t}^{I}(\omega) \nrightarrow 0$, this implies that:

$$
x_{s t} \rho_{s t} / w_{t-1} \rightarrow(1-c) p_{s z} / \theta_{z} \quad \text { a.s. }
$$

We can now show that uninformed traders are driven to extinction by the informed. Let us go back to the market clearing condition:

$$
\frac{x_{s t} \rho_{s t}}{w_{t-1}}=(1-c) \varepsilon_{t-1}^{I} \frac{p_{s z}}{\theta_{z}}+\left(1-\varepsilon_{t-1}^{I}\right) p_{s t}^{U}
$$

Because of no full revelation $p_{s t}^{U} \nrightarrow(1-c) p_{s z} / \theta_{z}$ and therefore necessarily $\varepsilon_{t}^{U} \rightarrow 0$ a.s.

\section{References}

[1] Allen, Beth, 1981, Generic Existence of Completely Revealing Equilibria for Economies with Uncertainty when Prices Convey Information, Econometrica 49, 1173-1199.

[2] Berk, Jonathan B., 1997, The Acquisition of Information in a Dynamic Market, Economic Theory 9, 441-451.

[3] Bias, Bruno and Raphael Shadur, 1997, Darwinian Selection does not Eliminate Irrational Traders, mimeo.

[4] Blume, Lawrence E., Margaret M. Bray and David Easley, 1982, Introduction to the Stability of Rational Expectations Equilibrium, Journal of Economic Theory 26, 313317.

[5] Blume, Lawrence E. and David Easley, 1982, Learning to Be Rational, Journal of Economic Theory 26, 340-351.

[6] Blume, Lawrence E. and David Easley, 1992, Evolution and Market Behaviour, Journal of Economic Theory 58, 9-40.

[7] Bray, Margaret M., 1981, Futures Trading, Rational Expectations, and the Efficient Market Hypothesis, Econometrica 49, 575-598. 
[8] Bray, Margaret M., 1982, Learning, Estimation, and the Stability of Rational Fxpectations, Journal of Economic Theory 26, 318-339.

[9] Colinsk, John, 1980, Costly Optimizers versus Cheap Imitators, Journal of Economic Behavior and Organization 1, 275-293.

[10] Cramer, Jan S., 1989, Econometric Applications of Maximum Likelihood Methods, Cambridge University Press, Cambridge.

[11] De Long, J. Bradford, Andrei Shleifer, Lawrence H. Summers and Robert J. Waldmann, 1990, Noise Trader Risk in Financial Markets, Journal of Political Economy 98, 703-738.

[12] De Long, J. Bradford, Andrei Shleifer, Lawrence H. Summers and Robert J. Waldmann, 1991, The Survival of Noise Traders in Financial Markets, Journal of Business 64, 1-19.

[13] Diamond, Douglas W. and Robert E. Verrecchia, 1981, Information Aggregation in a Noisy Expectations Economy, Journal of Financial Economics 9, 221-235.

[14] Dow, James and Rohit Rahi, 1997, Informed Trading, Investment and Welfare, European University Institute Working Paper 3/97.

[15] Fama, Eugene F., 1970, Efficient Capital Markets: A Review of Theory and Empirical Work, Journal of Finance 25, 383-417.

[16] Freedman, David A., 1975, On Tail Probabilities for Martingales, Annals of Probability $3,100-118$.

[17] Black, Fischer, 1986, Noise, Journal of Finance 3, 529-543.

[18] Friedman, Milton, 1953, The Case for Flexible Exchange Rates, in Essays in Positive Economics, University Chicago Press, Chicago.

[19] Green, Jerry R., 1977, The Non-Existence of Informational Equilibria, Review of Economic Studies 44, 451-464.

[20] Grossman, Sanford J., 1977, The Existence of Future Markets, Noisy Rational Fxpectations and Informational Externalities, Review of Economic Studies 64, 431-49.

[21] Grossman, Sanford J. and Joseph E. Stiglitz, 1980, On the Impossibility of Informationally Efficient Markets, American Economic Review 70, 393-408.

[22] Hellwig, Martin F., 1980, On the Aggregation of Information in Competitive Markets, Journal of Economic Theory 22, 477-498.

[23] Hirshleifer, Jack, 1971, The Private and Social Value of Information and the reward of Inventive Activity, American Economic Review 61, 561-574.

[24] Jordan, James S., 1982, The Generic Fxistence of Rational Expectations Equilibrium in the Higher Dimensional Case, Journal of Economic Theory 26, 224-243. 
[25] Jordan, James S. and Roy Radner, 1982, Rational Expectations in Microeconomic Models: An Overview, Journal of Economic Theory 26, 201-223.

[26] Kreps, David M., 1977, A Note on "Fulfilled Expectations" Equilibria, Journal of Economic Theory 14, 32-43.

[27] Kyle, Albert S. and F. Albert Wang, 1997, Speculation Duopoly with Agreement to Disagree: Can Overconfidence Survive the Market Test?, Journal of Finance 5, 20732090.

[28] Laffont, Jean-Jacques, 1985, On the Welfare Analysis of Rational Fxpectations Equilibria with Asymmetric Information, Econometrica 53, 1-29.

[29] Lucas, Robert E., 1972, Fxpectations and the Neutrality of Money, Journal of Economic Theory 4, 103-124.

[30] Radner, Roy, 1979, Rational Expectations Equilibrium: Generic Existence and the Information Revealed by Prices, Econometrica 47, 655-678.

[31] Rogers, Alan R., 1988, Does Biology Constrain Culture?, American Anthropologist 90, 819-831.

[32] Rudin, Walter, 1976, Principles of Mathematical Analysis, McGraw-Hill International Editions.

[33] Sandroni, Alvaro, 1997, Do Markets Favour Agents Able to Make Accurate Predictions?, Kellogg Graduate School of Management, mimeo.

[34] Sciubba, Emanuela, 1998, The Evolution of Portfolio Rules and the Capital Asset Pricing Model, University of Cambridge, mimeo.

[35] Shefrin, Hersh and Meir Statman, 1994, Behavioral Capital Asset Pricing Theory, Journal of Financial and Quantitative Analysis 29, 323-349.

[36] Sunder, Shyam, 1992, Market for Information: Experimental Evidence, Econometrica $60,667-695$.

[37] Stein, Jeremy C., 1987, Informational Externalities and Welfare-reducing Speculation, Journal of Political Economy 95, 1123-1145.

[38] Verrecchia, Robert E., 1982, Information Acquisition in a Noisy Rational Expectations Economy, Econometrica 50, 1415-1430. 